\title{
The $\mathbb{G}_{m}$-equivariant motivic cohomology of Stiefel varieties
}

\author{
BEN WiLLIAMS
}

\begin{abstract}
We derive a version of the Rothenberg-Steenrod, fiber-to-base, spectral sequence for cohomology theories represented in model categories of simplicial presheaves. We then apply this spectral sequence to calculate the equivariant motivic cohomology of $\mathrm{GL}_{n}$ with a general $\mathbb{G}_{m}$-action; this coincides with the equivariant higher Chow groups. The motivic cohomology of $\mathrm{PGL}_{n}$ and some of the equivariant motivic cohomology of a Stiefel variety, $V_{m}\left(\mathbb{A}^{n}\right)$, with a general $\mathbb{G}_{m}$-action is deduced as a corollary.
\end{abstract}

19E15; 14C15, 18G55

\section{Introduction}

Let $k$ be a field, not necessarily algebraically closed or of characteristic 0 . Let $G$ be a linear algebraic group acting on a variety $X$. The equivariant higher Chow groups of a variety $X$ are defined in general by Edidin and Graham in [5], developing an idea presented in [19] where the classifying space of $G$ is constructed. Subsequent calculations of equivariant Chow groups have been carried out especially in the important cases of $A_{G}^{*}$, as by Vistoli in [20], or in order to calculate the ordinary Chow groups of the moduli spaces $\mathcal{M}_{g}$ of genus $g$ curves, [5, Appendix by A. Vistoli]. The chief tool used in these calculations has been the equivariant stratification of varieties, and they are algebro-geometric in nature. The higher equivariant Chow groups have not been much computed.

In this paper we adopt the view that the equivariant higher Chow groups should behave much like Borel equivariant singular cohomology groups, at least when the groups and varieties concerned are without arithmetic complications, and therefore methods of $\mathbb{A}^{1}$-homotopy may be employed. There are a great many restrictions on $X$ and $G$ that limit the applicability of this idea, but in the special case where $X$ is a Stiefel variety, $V_{m}\left(\mathbb{A}^{n}\right)$, parametrizing full rank $n \times m$-matrices and where $G=\mathbb{G}_{m}$, or more generally $G$ is a torus, the restrictive criteria are all satisfied, and we may deduce some of $\mathrm{CH}_{G}^{*}(X, *)_{F}$ (where $F$ is a field of coefficients) from $\mathrm{CH}^{*}(X, *)_{F}$. This is done in Corollary 2.17.1. 
We may identify the Stiefel variety $V_{m}\left(\mathbb{A}^{n}\right)$ as the space of matrices representing injective linear maps $A: k^{m} \rightarrow k^{n}$ where both source and target are equipped with a standard basis. By assigning the basis elements $\mathbb{Z}$-gradings, say $u_{1}, \ldots, u_{m}$ and $v_{1}, \ldots, v_{n}$ to be specific, we endow the source and target with the structure of a $\mathbb{G}_{m}$-representation. Consequently, if we let $\mathbb{G}_{m}$ act on $V_{m}\left(\mathbb{A}^{n}\right)$ by

$$
z \cdot A=\left(\begin{array}{cccc}
z^{-u_{1}} & & & \\
& z^{-u_{2}} & & \\
& & \ddots & \\
& & & z^{-u_{m}}
\end{array}\right) A\left(\begin{array}{cccc}
z^{v_{1}} & & & \\
& z^{v_{2}} & & \\
& & \ddots & \\
& & & z^{v_{n}}
\end{array}\right),
$$

we incorporate the $\mathbb{Z}$-grading into the structure of $V_{m}\left(\mathbb{A}^{n}\right)$ in the following sense: if $R$ is a $\mathbb{Z}$-graded $k$-algebra, then Spec $R$ is a $k$-scheme equipped with a $\mathbb{G}_{m}$-action, and a $\mathbb{G}_{m}$-equivariant map Spec $R \rightarrow V_{m}\left(\mathbb{A}^{n}\right)$ classifies a graded, full rank map $R^{m} \rightarrow R^{n}$. In the special case where $n=m$, where the source of the linear map is entirely in degree 0 and the target entirely in degree 1 , we recover as a quotient the scheme $\mathrm{PGL}_{n}$, and we present the higher Chow groups of PGL $n$ in Corollary 2.15.1.

The structure of the paper is as follows. The first section is written in general language, applying to an unspecified cohomology theory satisfying certain properties and representable in some model structure on a category of simplicial presheaves on a category C. In Proposition 1.6 a spectral sequence is used to compute the cohomology of a bar complex $B(G, X)$, which is a version of the Borel equivariant cohomology of $X$ with respect to the $G$-action. If the cohomology of $G$ and $X$ are particularly well-behaved, this spectral sequence admits description on the $\mathrm{E}_{2}$-page, as described in Theorem 1.7.

In Theorem 1.15, the case of a free action is treated, describing a quotient in $\mathbf{C}$, say $X=Y / G$, in terms of the cohomology of $Y$ and $G$, provided a battery of conditions on $Y$ and $G$ are satisfied, and provided the quotient $X=Y / G$ satisfies a local triviality condition. This is an analogue of a spectral sequence in classical algebraic topology that goes by several names, among them "Rothenberg-Steenrod" and "fiber-to-base Eilenberg-Moore"; it appears in Rothenberg and Steenrod [17].

The second section then specializes to the case of motivic cohomology, and to our form of Borel equivariant motivic cohomology. We argue in Proposition 2.6 that under the hypotheses that are satisfied throughout the paper, chiefly that $G$ be a special group, that this Borel equivariant motivic cohomology is isomorphic after reindexing to the equivariant higher Chow groups of [5]. We employ the motivic cohomological indexing throughout. Some facts on the ordinary motivic cohomology of GL $n$ and related varieties are recalled, before we establish a sequence of technical results, always arguing 
about differentials in spectral sequences, culminating in a nearly complete description of a convergent spectral sequence calculating the $\mathbb{G}_{m}$-equivariant motivic cohomology of $\mathrm{GL}_{n}$ for a general $\mathbb{G}_{m}$-action on both the left and right, in Proposition 2.16. In the final section, we deduce what we can of the analogous spectral sequence for a general action of $\mathbb{G}_{m}$ on $V_{m}\left(\mathbb{A}^{n}\right)$. Some technicalities on homological algebra, which are required for the description of the $\mathrm{E}_{2}$-pages of the spectral sequences, are included in the appendix.

\section{Spectral sequences for bisimplicial sheaves}

\subsection{Spaces}

In the first section we work in some generality. We suppose $\mathbf{C}$ is a site, with topology $\tau$. As a technical convenience we assume $\mathbf{C}$ has enough points. We think of the category of simplicial sheaves, $s \mathbf{S h}_{\tau}(\mathbf{C})$, on $\mathbf{C}$ as being a category of spaces, and it is here that we carry out the bulk of our homotopy theory.

There are several model structures on the categories $s \mathbf{S h}_{\tau}(\mathbf{C})$. Among them are various global model structures, where weak equivalences are detected objectwise, and various local model structures, where the weak equivalences $\mathcal{F} \rightarrow \mathcal{H}$ are the maps of simplicial presheaves that yield weak equivalences at all points $p^{*}$ of the site $\mathbf{C}$. We choose as our model structures of preference the injective model structures where the cofibrations are the maps $\mathcal{F} \rightarrow \mathcal{H}$ which are monomorphisms of simplicial sheaves. The fibrations are thus determined as the maps that satisfy a lifting property with respect to trivial cofibrations. By the global model structure or the local model structure we mean the global injective and local injective model structure respectively. The injective local model structure originates with Joyal, and is presented by Jardine in [10].

We endow $s \mathbf{S h}_{\tau}(\mathbf{C})$ with a model structure, $M$, which is a left Bousfield localization of the injective local model structure. The best-known examples of such model structures are the injective model structures themselves, especially for the étale topology, and the $\mathbb{A}^{1}$-model structure of Morel and Voevodsky [14]. In a sop to excessive generality, we do not require that representable presheaves be sheaves on $\mathbf{C}$. We shall write 'global weak equivalence', 'injective weak equivalence' in the global or local cases; the bare term 'weak equivalence', when applied to sheaves or presheaves, will mean ' $M$-weak equivalence'.

We remark that in addition to there being an injective local model structure on $s \mathbf{S h}(\mathbf{C})$, there is an injective $\tau$-local model structure on $s \operatorname{Pre}(\mathbf{C})$, where the weak equivalences are again detected on stalks, so that the natural transformation from the identity to the 
associated sheaf functor $F \mapsto a(F)$ is always a weak equivalence. The distinction between the injective model structure on $s \mathbf{S h}_{\tau}(\mathbf{C})$ and that defined on $s \mathbf{P r e}(\mathbf{C})$ is nugatory.

We observe that the local injective structure on $s \mathbf{S h}_{\tau}(\mathbf{C})$ is in fact a localization of the global structure, see for instance Isaksen [9]. In particular, maps which are weak equivalences in the global model category will be weak equivalences in all the model categories under consideration.

Both $s \mathbf{S h}_{\tau}(\mathbf{C})$ and $s \mathbf{P r e}(\mathbf{C})$, with any one of the previously mentioned model structures, have associated pointed structures, the underlying categories of which will be denoted by $s \mathbf{S h}_{\tau}(\mathbf{C})_{+}$and $s \mathbf{P r e}(\mathbf{C})_{+}$.

\subsection{Cohomology}

We fix a bigraded representable cohomology theory $L^{*, *}$, which is to say a bigraded family of fibrant objects $L(p, q) \in s \mathbf{S h}_{\tau}(\mathbf{C})_{+}$such that

$$
L^{p, q}(X)=\left[X_{+}, L(p, q)\right]
$$

in $s \mathbf{S h}(\mathbf{C})_{+}$. We demand that there be $M$-weak equivalences $L(p, q) \rightarrow \Omega L(p+1, q)$, so that there is a suspension isomorphism $L^{p+r, q}\left(\Sigma^{r} X\right) \cong L^{p, q}(X)$, from which it follows that $L^{p, q}(\cdot)$ is an abelian group valued functor. We require finally that there be multiplication maps $L(p, q) \wedge L\left(p^{\prime}, q^{\prime}\right) \rightarrow L\left(p+p^{\prime}, q+q^{\prime}\right)$ satisfying the usual diagrammatic conditions, endowing $L^{p, q}(X)$ with a functorial ring structure, which we require to be graded commutative in the first grading and strictly commutative in the second.

We are essentially demanding that $L(\cdot, q)$ be a ring object in a category of graded $S^{1}$-spectra, and we might have presented the above requirements in the context of some graded stable model category. We choose not to do so, since the cost of having to pass from unstable to stable model structures outweighs the benefit of streamlined arguments in the stable setting.

If $X$ is an object in $s \mathbf{S h}_{\tau}(\mathbf{C})_{+}$, we write $\widetilde{L}^{p, q}=[X, L(p, q)]$.

Theories meeting the criteria we demand of $L(p, q)$ abound. The application of the general theory in the sequel will be to the case of $L(p, q)=\mathbb{H} R(p, q)$, the motivic Eilenberg-Mac Lane spaces representing $R$-valued motivic cohomology, see Voevodsky [22] for instance, in the $\mathbb{A}^{1}$-model structure on $s \mathbf{S h}_{N i s}(\mathbf{S m} / k)$. Other examples include, for various choices of $\mathbf{C}$ and $M$, algebraic $K$-theory and étale cohomology. 
Let $\mathcal{A}$ be a collection of objects of $s \mathbf{S h}_{\tau}(\mathbf{C})$. We say $L^{*, *}(\cdot)$ is bounded below on $\mathcal{A}$ if it meets the following condition: for all integers $q$, there exists some $c(q)$ such that $L^{p, q}(X)=0$ for all $X \in \mathcal{A}$ whenever $p<c(q)$. If $\mathcal{A}=s \mathbf{S h}_{\tau}(\mathbf{C})$, we simply say $L^{*, *}$ is bounded below. Unfortunately, the boundedness of motivic cohomology is essentially the Beilinson-Soulé vanishing conjecture, which is currently known only in some cases-for instance for $\mathrm{H}^{*, *}(\operatorname{Spec} F ; \mathbb{Z})$ where $F$ is either a finite field or a number field.

\subsection{Bisimplicial sheaves and cohomology}

Our references for simplicial homotopy theory are Hirschhorn [8, Chapter 18] and Bousfield and Kan [3]. Let $X_{\bullet}$ be a simplicial object in $s \mathbf{S h}_{\tau}(\mathbf{C})$ or $s \mathbf{P r e}(\mathbf{C})$, which one views either as a bisimplicial (pre)sheaf in $\Delta^{\mathrm{op}} \times \Delta^{\mathrm{op}} \rightarrow \mathbf{S h}(\mathbf{C})$ (in $\Delta^{\mathrm{op}} \times \Delta^{\mathrm{op}} \rightarrow \operatorname{Pre}(\mathbf{C})$ respectively), or as a $\Delta^{\mathrm{op}}$-shaped diagram in the category $s \mathbf{S h}_{\tau}(\mathbf{C})$ (or in the category $s$ Pre $(\mathbf{C})$ respectively). There are a diversity of methods of 'realizing' a simplicial object $X_{\bullet}$. In our model structures, where all objects are cofibrant, these result in identical, isomorphic, or at worst weakly equivalent objects.

A simplicial object $X \bullet$ in $s \mathbf{S h}_{\tau}(\mathbf{C})($ or $s \mathbf{P r e}(\mathbf{C}))$ is a $\Delta^{\mathrm{op}}$-shaped diagram

$$
X_{\bullet}: \Delta^{\mathrm{op}} \rightarrow s \mathbf{S h}_{\tau}(\mathbf{C})
$$

(or $X_{\bullet}: \Delta^{\text {op }} \rightarrow s \operatorname{Pre}(\mathbf{C})$ ). We define the realization $\left|X_{\bullet}\right|$ to be hocolim $\Delta^{\text {op }} X_{\bullet}$, using the explicit construction of hocolim as given in [8].

From the description given there it is clear that if $X_{\bullet}$ is a simplicial object in $s \mathbf{S h}_{\tau}(\mathbf{C})$, then it does not matter whether the realization or the homotopy colimit is taken in the category $s \mathbf{S h}_{\tau}(\mathbf{C})$ or $s \operatorname{Pre}(\mathbf{C})$, the answer in either case is the same. This applies indeed to arbitrary homotopy colimits of diagrams in the category $s \mathbf{S h}_{\tau}(\mathbf{C})$.

The $M$-model structure on $s \mathbf{S h}_{\tau}(\mathbf{C})$ inherits the cofibrations of the injective model structure, which are simply the monomorphisms of presheaves and which coincide with the monomorphisms of sheaves. It follows that if $\mathcal{D}$ is a diagram in $s \mathbf{S h}_{\tau}(\mathbf{C})$, then hocolim $\mathcal{D}$ has the same construction for the injective and the $M$-model structure.

We recall from [14] that a point of a site is a covariant functor that commutes with finite limits and all colimits. A map $f: X \rightarrow Y$ in $s \mathbf{S h}_{\tau}(\mathbf{C})$ or in $s \mathbf{P r e}(\mathbf{C})$ is an injective weak equivalence if and only if it induces a weak equivalence at all points. Let $\mathbf{I}$ be a small category and let $X$ be an $\mathbf{I}$-shaped diagram in $s \mathbf{S h}_{\tau}(\mathbf{C})$ (respectively in $s \mathbf{P r e}(\mathbf{C}))$. By construction of hocolim, one has

$$
p(\underset{\mathbf{I}}{\operatorname{hocolim}} X)=\underset{\mathbf{I}}{\operatorname{hocolim}} p X .
$$

This is given in [14, Lemma 2.1.20]. 
One can see directly from the construction of the functor $|\cdot|$ that it preserves colimits. If $X_{\bullet}$ and $Y_{\bullet}$ are two objects in $s s \mathbf{S h}_{\tau}(\mathbf{C})$, then the projections $\pi: X_{\bullet} \times Y_{\bullet} \rightarrow X_{\bullet}$, along with functoriality, imply there is a weak equivalence $\left|X_{\bullet} \times Y_{\bullet}\right| \rightarrow\left|X_{\bullet}\right| \times\left|Y_{\bullet}\right|$. In the pointed case, the preservation of colimits implies that there is an induced map $\left|X_{\bullet} \wedge Y_{\bullet}\right| \rightarrow\left|X_{\bullet}\right| \wedge\left|Y_{\bullet}\right|$.

Although one can impose a model structure on ${ }_{s s} \mathbf{S h}_{\tau}(\mathbf{C})$, we do not do so. If $D: \mathcal{I} \rightarrow{ }_{s s} \mathbf{S h}_{\tau}(\mathbf{C})$ is a diagram (a functor from a small category) then we denote by $\operatorname{hocolim}_{I} D(i)$ the termwise homotopy colimit, that is, the object in ${ }_{s s} \mathbf{S h}_{\tau}(\mathbf{C})$ given by the functor $n \mapsto \operatorname{hocolim}_{i} D(i)_{n}$.

Proposition 1.1 Let $D: \mathcal{I} \rightarrow s s \mathbf{S h}_{\tau}(\mathbf{C})$ be a diagram in the category of simplicial spaces. One has $M-$ weak equivalences

$$
|\underset{i \in \mathbf{I}}{\operatorname{hocolim}} D(i)| \simeq \underset{i \in \mathbf{I}}{\operatorname{hocolim}}|D(i)| .
$$

Proof We will show these are injective weak equivalences, a fortiori that they are $M$-weak equivalences. The given equivalence amounts to

$$
\underset{\Delta^{\mathrm{op}}}{\operatorname{hoc}} \underset{i \in \mathbf{I}}{\operatorname{hocolim}} D(i) \simeq \text { injective } \underset{i \in \mathbf{I}}{\operatorname{hocolim}} \underset{\Delta^{\mathrm{op}}}{\operatorname{hocolim}} D(i),
$$

which is a consequence of the Fubini theorem for hocolim, [3, Chapter XIII, 3.3], proved there for simplicial sets, which can be promoted to the current setting by arguing at points.

The following is a special case of [8, Theorem 18.5.3].

Proposition 1.2 If $\mathbf{I}$ is a small category and $f: X \rightarrow Y$ is a natural transformation of I-shaped diagrams in $s \mathbf{S h}_{\tau}(\mathbf{C})$ which is an objectwise $M$-weak equivalence then the induced map hocolim $X \rightarrow$ hocolim $_{\mathbf{I}} Y$ is an $M$-weak equivalence. In particular, if $f: X_{\bullet} \rightarrow Y_{\bullet}$ is an objectwise $M$-weak equivalence of simplicial objects in $s \mathbf{S h}_{\tau}(\mathbf{C})$, then $\left|X_{\bullet}\right| \rightarrow\left|Y_{\bullet}\right|$ is an $M$-weak equivalence.

We denote by pt the constant sheaf with value the one point simplicial set. We denote the $L$-cohomology of pt by $\mathbb{L}=L^{*, *}(\mathrm{pt})$; it is a bigraded ring. The $L$-cohomology of any space is a module over $\mathbb{L}$.

Suppose $A_{\bullet}$ is a cosimplicial abelian group. There are two associated chain complexes of abelian groups, the first of which is the naïve $A_{*}$, in which the terms are precisely the $A_{p}$ and the differentials are alternating sums of the coface maps, the second is the normalized chain complex $N_{*}\left(A_{\bullet}\right)$, where $N_{p}\left(A_{\bullet}\right)$ is the subgroup of nondegenerate cochains in $A_{p}$. It is well-known that the inclusion $N_{*}\left(A_{\bullet}\right) \rightarrow A_{*}$ is a quasi-isomorphism. 
The following proposition allows us to compute the $L$-cohomology of the realization of a simplicial object in $s \mathbf{S h} \mathbf{h}_{\tau}(\mathbf{C})$ provided issues of convergence can be resolved.

Proposition 1.3 Suppose $X_{\bullet}$ is a simplicial object in $s \mathbf{S h}_{\tau}(\mathbf{C})$. Application of $L^{*, *}(\cdot)$ produces a cosimplicial bigraded abelian group. Write $N_{*}\left(L^{*, *}\left(X_{\bullet}\right)\right)$ for the associated normalized cochain complex.

There is a trigraded spectral sequence

$$
\mathrm{E}_{1}^{p, q, *}=N_{p}\left(L^{q, *}\left(X_{\bullet}\right)\right) \Longrightarrow \lim _{s \rightarrow \infty} L^{p+q, *}\left(\left|\mathrm{sk}_{s} A \bullet\right|\right),
$$

converging conditionally. The spectral sequence is functorial in both $X_{\bullet}$ and $L^{*, *}$. The differentials on the $r$-th page take the form $d_{r}: \mathrm{E}_{r}^{p, q} \rightarrow \mathrm{E}_{r}^{p+r, q-r+1}$. Additionally, the differential on the $\mathrm{E}_{1}$-page coincides with the differential on $N_{*}\left(L^{*, *}\left(X_{\bullet}\right)\right)$, so the $\mathrm{E}_{2}-$ page may be identified with the homology of the complex $L^{*, *}\left(X_{q-1}\right) \rightarrow L^{*, *}\left(X_{q}\right) \rightarrow$ $L^{*, *}\left(X_{q+1}\right)$.

Suppose now that $X_{\bullet}$ and $Y_{\bullet}$ are two simplicial objects in $s \mathbf{S h}_{\tau}(\mathbf{C})$. Then there is a natural pairing of spectral sequences inducing products

$$
\mathrm{E}_{r}^{p, q}\left(X_{\bullet}\right) \otimes \mathrm{E}_{r}^{p^{\prime}, q^{\prime}}\left(Y_{\bullet}\right) \rightarrow \mathrm{E}_{r}^{p+p^{\prime}, q+q^{\prime}}\left(X_{\bullet} \times Y_{\bullet}\right)
$$

with respect to which the differentials are derivations.

Proof The third grading does not play much part in this proof, and we suppress it.

If $X_{\bullet}$ is an object in $\operatorname{ssSh}_{\tau}(\mathbf{C})$, then $L^{*, *}\left(X_{\bullet}\right)$ will be used to denote the cosimplicial object $n \mapsto L^{*, *}\left(X_{n}\right)$.

The bulk of our proof amounts to little more than the observation that the argument of Segal in [18, Proposition 5.1] carries over to the present setting. Where [18] has pairs $(X, Y)$, we have cofibers $\operatorname{cofib}(Y \rightarrow X)$; the other notational discrepancies between that paper and this one are minor.

The simplicial object $X_{\bullet}$ is filtered by the skeleta, $\operatorname{sk}_{i}\left(X_{\bullet}\right)$. Write $B_{i}$ for the cofiber

$$
\left|\mathrm{sk}_{i-1}\left(X_{\bullet}\right)\right| \stackrel{\iota_{i}}{\longrightarrow}\left|\mathrm{sk}_{i}\left(X_{\bullet}\right)\right| \longrightarrow B_{i}
$$

Since $\iota_{i}$ is an inclusion, it follows that $B_{i}=\left|\mathrm{sk}_{i}\left(X_{\bullet}\right)\right| /\left|\mathrm{sk}_{i-1}\left(X_{\bullet}\right)\right|$. There is a global weak equivalence

$$
B_{i} \simeq_{\text {global }} \Sigma^{i}\left(\operatorname{cofib}\left(X_{i}^{\mathrm{deg}} \rightarrow X_{i}\right)\right)
$$

where $X_{i}^{\text {deg }}$ denotes the image of the degeneracies in $X^{i}$. 
The filtration by skeleta leads, as by Boardman in [2], to an unrolled exact couple:

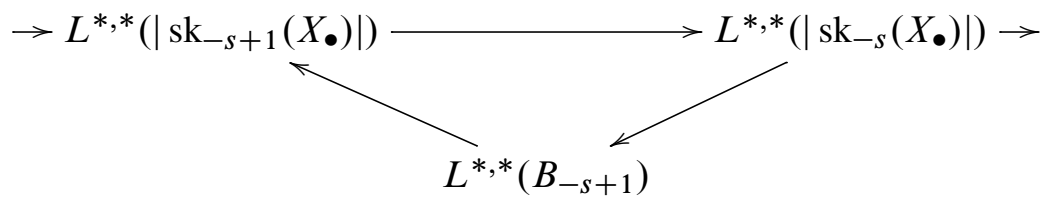

This gives rise to a spectral sequence. Using the indexing $\mathrm{E}_{1}^{p, q}=L^{q, *}\left(B_{-p}\right)$, the construction yields differentials of the form

$$
d_{r}: \mathrm{E}_{r}^{p, q} \rightarrow \mathrm{E}_{r}^{p+r, q-r+1},
$$

as claimed.

The arguments of [18] apply, in particular the identification of the $\mathrm{E}_{1}$-page, including the differentials, with the reduced chain complex associated to the cosimplicial abelian group $L^{*, *}\left(X_{\bullet}\right)$.

We note that $L^{*, *}\left(\left|\mathrm{sk}_{-s}\left(X_{\bullet}\right)\right|\right)=0$ for $s>0$, so that

$$
\lim _{s} L^{*}\left(\left|\mathrm{sk}_{-s}\left(X_{\bullet}\right)\right|\right)=\operatorname{Rlim}_{s} L^{*}\left(\left|\mathrm{sk}_{-s}\left(X_{\bullet}\right)\right|\right)=0,
$$

and in the terminology of [2], we immediately have conditional convergence of the spectral sequence to

$$
\lim _{s \rightarrow-\infty} L^{*, *}\left(\left|\mathrm{sk}_{-s}\left(X_{\bullet}\right)\right|\right)=L^{*, *}\left(\left|\mathrm{sk}_{-s}\left(X_{\bullet}\right)\right|\right) .
$$

Suppose $X_{\bullet}, Y_{\bullet}$ are two objects in $s s \mathbf{S h}_{\tau}(\mathbf{C})$. For any nonnegative integers $s, s^{\prime}$, there are Eilenberg-Zilber maps $\operatorname{sk}_{s+s^{\prime}}\left(X_{\bullet} \times Y_{\bullet}\right) \rightarrow \operatorname{sk}_{S}\left(X_{\bullet}\right) \times \operatorname{sk}_{s^{\prime}}\left(Y_{\bullet}\right)$; we combine the realization of this map with $\left|\operatorname{sk}_{S}\left(X_{\bullet}\right) \times \operatorname{sk}_{s^{\prime}}\left(Y_{\bullet}\right)\right| \rightarrow\left|\mathrm{sk}_{S}\left(X_{\bullet}\right)\right| \times\left|\mathrm{sk}_{s^{\prime}}\left(Y_{\bullet}\right)\right|$ and so obtain a pairing

$$
\widetilde{L}^{*, *}\left(\left|\mathrm{sk}_{S} X_{\bullet}\right|\right) \otimes \widetilde{L}^{*, *}\left(\left|\mathrm{sk}_{S^{\prime}} Y_{\bullet}\right|\right) \rightarrow \widetilde{L}^{*, *}\left(\left|\operatorname{sk}_{s+s^{\prime}}\left(X_{\bullet} \times Y_{\bullet}\right)\right|\right)
$$

This product gives rise in the usual way to a pairing on the $\mathrm{E}_{2}$-page, and it is standard that all differentials $d_{r}: \mathrm{E}_{r} \rightarrow \mathrm{E}_{r}$ are derivations with respect to this product, so that the product persists to the $\mathrm{E}_{\infty}$-page.

Corollary 1.3.1 Suppose $L^{*, *}(\cdot)$ is bounded below on the set $\left\{X_{S}\right\}_{s=0}^{\infty}$. The spectral sequence of the previous proposition converges to $L^{p+q, *}\left(\left|X_{\bullet}\right|\right)$. In each weight $r$, one has

$$
\mathrm{E}_{1}^{p, q, r}=N_{p}\left(L^{q, r}\left(X_{\bullet}\right)\right) \Longrightarrow L^{p+q, r}\left(\left|X_{\bullet}\right|\right)
$$


Proof Write $\iota_{s}$ for the map $\iota_{s}:\left|\mathrm{sk}_{-s}\left(A_{\bullet}\right)\right| \rightarrow\left|\mathrm{sk}_{-s+1}\left(A_{\bullet}\right)\right|$. Since $\iota_{n}$ is a cofibration for all $n$, one has

$$
\left|A_{\bullet}\right|=\underset{S}{\operatorname{colim}}\left|A_{S}\right|=\underset{S}{\operatorname{hocolim}}\left|A_{S}\right|=\operatorname{tel}\left|A_{S}\right|,
$$

where tel denotes the mapping telescope construction. A standard argument, as by May in [12, Chapter 19.4], gives

$$
L^{*, *}\left(\left|A_{\bullet}\right|\right) \cong \lim _{s} L^{*, *}\left(\left|\mathrm{sk}_{-s}\left(A_{\bullet}\right)\right|\right),
$$

contingent on the vanishing of the Rlim-term in

$$
\begin{aligned}
& 0 \rightarrow \lim _{s} L^{*, *}\left(\left|\mathrm{sk}_{-s}\left(A_{\bullet}\right)\right|\right) \rightarrow \prod_{s=0}^{\infty} L^{*, *}\left(\left|\mathrm{sk}_{S}\left(A_{\bullet}\right)\right|\right) \\
& \stackrel{\mathrm{id}-\iota^{*}}{\longrightarrow} \prod_{s=0}^{\infty} L^{*, *}\left(\left|\mathrm{sk}_{S}\left(A_{\bullet}\right)\right|\right) \rightarrow \underset{s}{\operatorname{Rim}} L^{*, *}\left(\left|\mathrm{sk}_{-s}\left(A_{\bullet}\right)\right|\right) \rightarrow 0
\end{aligned}
$$

By restricting to particular bidegrees, $L^{p, q}(\cdot)$, we obtain

$$
\begin{aligned}
0 \rightarrow \lim _{s} L^{p, q}\left(\left|\mathrm{sk}_{-s}\left(A_{\bullet}\right)\right|\right) & \rightarrow \prod_{s=0}^{\infty} L^{p, q}\left(\left|\mathrm{sk}_{S}\left(A_{\bullet}\right)\right|\right) \\
\stackrel{\mathrm{id}-\iota^{*}}{\longrightarrow} & \prod_{s=0}^{\infty} L^{p, q}\left(\left|\mathrm{sk}_{S}\left(A_{\bullet}\right)\right|\right) \rightarrow \operatorname{Rlim}_{s} L^{p, q}\left(\left|\mathrm{sk}_{-}\left(A_{\bullet}\right)\right|\right) \rightarrow 0
\end{aligned}
$$

but since the map $\iota_{i}^{*}: L^{p, q}\left(\left|\operatorname{sk}_{i}\left(A_{\bullet}\right)\right|\right) \rightarrow L^{p, q}\left(\left|\operatorname{sk}_{i-1}\left(A_{\bullet}\right)\right|\right)$ is an isomorphism when

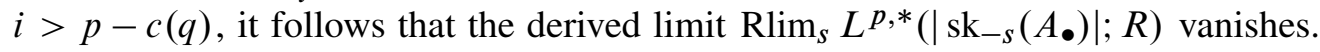
Consequently,

$$
L^{*, *}\left(\left|A_{\bullet}\right|\right) \cong \lim _{S} L^{*, *}\left(\left|\mathrm{sk}_{-S}\left(A_{\bullet}\right)\right|\right)
$$

as required.

The convergence is strong: since $\mathrm{E}_{1}^{p, q, r}=0$ if $q<c(r)$, each group can support at most finitely many differentials.

\subsection{The bar construction}

Suppose $G$ is a group object in $s \mathbf{S h}_{\tau}(\mathbf{C})$ and $X, Y \in s \mathbf{S h}_{\tau}(\mathbf{C})$ admit right- and leftactions by $G$ respectively. We can form the two-sided bar construction $B(X, G, Y)$. by precisely the formulas of [11]. It is a simplicial object in $s \mathbf{S h}_{\tau}(\mathbf{C})$, ie a bisimplicial 
sheaf, but we suppress the simplicial indices arising from the intrinsic structure of objects in $s \mathbf{S h}_{\tau}(\mathbf{C})$. One has

$$
B(X, G, Y)_{n}=X \times \overbrace{G \times \cdots \times G}^{n \text {-times }} \times Y .
$$

Bar constructions exhibit a wealth of desirable properties in ordinary homotopy theory, for which see May [11], and some of these results also hold in the context of the homotopy of sheaves. We shall frequently prefer to work with an object of $s \mathbf{S} \mathbf{h}_{\tau}(\mathbf{C})$, rather than a simplicial object, so we adopt the notational convention that $B(X, G, Y)=|B(X, G, Y) \bullet|$.

Proposition 1.4 The constructions $B(X, G, Y)_{\bullet}, B(X, G, Y)$ are natural in all three variables, in the sense that, if $G \rightarrow G^{\prime}$ is a homomorphism of group objects, and if $X, Y$ are right- and left- $G$-spaces, and $X^{\prime}$ and $Y^{\prime}$ are right- and left- $G^{\prime}$-spaces, such that there are maps $X \rightarrow X^{\prime}$ and $Y \rightarrow Y^{\prime}$ of right- and left- $G^{\prime}$-spaces, then there is a map $B(X, G, Y)_{\bullet} \rightarrow B\left(X^{\prime}, G^{\prime}, Y^{\prime}\right)_{\bullet}$, and similarly for the realization.

Proposition 1.5 Suppose that the model structure $M$ has the property that if $A \rightarrow A^{\prime}$ and $B \rightarrow B^{\prime}$ are weak equivalences, then $A \times B \rightarrow A^{\prime} \times B^{\prime}$ is a weak equivalence. In the notation of the previous proposition, if $G \rightarrow G^{\prime}, X \rightarrow X^{\prime}$ and $Y \rightarrow Y^{\prime}$ are weak equivalences, then $B(X, G, Y) \rightarrow B\left(X^{\prime}, G^{\prime}, Y^{\prime}\right)$ is a weak equivalence.

Proof This is straightforward levelwise, and the passage to realizations is effected by Proposition 1.2.

We observe that the condition of the previous proposition on the model structure $M$ is met by $\tau$-local model structures, or by the $\mathbb{A}^{1}$-local model structure of [14].

We remark also that for any $U \in \mathbf{C}$, and $X, G, Y$ as before, one has the identity

$$
B(X, G, Y)(U)=B(X(U), G(U), Y(U)) .
$$

Given an object $Y$ on which $G$ acts on the left, we define a Borel construction on $Y$ by $B(\mathrm{pt}, G, Y)=B(G, Y)$. Our construction is functorial in both $Y$ and $G$.

Proposition 1.6 Suppose $G$ is a group object in $s \mathbf{S h}_{\tau}(\mathbf{C})$ acting on the left on $Y \in s \mathbf{S h}_{\tau}(\mathbf{C})$. There is a spectral sequence of algebras

$$
\mathrm{E}_{1}^{p, q}=N_{p}\left(L^{q, *}(B(G, Y) \bullet)\right),
$$

which is natural in both $G$ and $Y$, in that a map $(G, Y) \rightarrow\left(G^{\prime}, Y^{\prime}\right)$ induces a map of spectral sequences. 
Suppose that $L^{*, *}$ is bounded below on the set $\left\{B(G, Y)_{s}\right\}_{s=0}^{\infty}$, then the spectral sequence in question converges strongly:

$$
\mathrm{E}_{1}^{p, q}=N_{p}\left(L^{q, *}(B(G, Y) \bullet)\right) \Rightarrow L^{p+q, *}(B(G, Y)) .
$$

Proof We apply Proposition 1.3 and Corollary 1.3.1 to the case of $B(G, Y)$.

Of course, everything goes through equally well if the $G$-action is on the left, and we obtain the construction $B(X, G, \mathrm{pt})$. We understand all subsequent results in this section as asserting also the equivalent result for such an action mutatis mutandis.

\subsection{Objects having finitely generated projective cohomology}

Recall that we write $\mathbb{L}$ for the cohomology $L^{*, *}(\mathrm{pt})$.

We encapsulate all the good behavior we typically demand of objects in an omnibus definition.

Definition 1 If an object $Y$ is well-behaved in the sense that $L^{*, *}(Y)$ is finitely generated projective $\mathbb{L}$-module, and if a Künneth isomorphism obtains

$$
L^{*, *}(Y) \otimes_{\mathbb{L}} L^{*, *}(X) \cong L^{*, *}(Y \times X)
$$

for all objects $X$, then we say $Y$ is $\mathbb{L}$-projective.

The Künneth spectral sequence

$$
\operatorname{Tor}^{\mathbb{L}}\left(L^{*, *}(X), L^{*, *}(Y)\right) \Longrightarrow L^{*, *}(X \times Y)
$$

need not exist in general, but if it does, and if $L^{*, *}(Y)$ is projective over $\mathbb{L}$, then it is degenerate and the Künneth isomorphism holds.

Especially valuable to us are group objects $G$ that are $\mathbb{L}$-projective. In the case of motivic cohomology defined on the $\mathbb{A}^{1}$-model structure on $s \mathbf{S h}_{N i s}(\mathbf{S m} / k)$-the setting that eventually will occupy our full attention—examples of such group objects include finite groups, $\mathrm{GL}_{n}$ (for which see Dugger and Isaksen [4] and Theorem 2.7), $\mathrm{SL}(n)$ and finite products of these groups.

For any $\mathbb{L}$-module, $N$, we use the notation $\hat{N}$ for $\operatorname{Hom}_{\mathbb{L}}(N, \mathbb{L})$. For a projective $\mathbb{L}$-module of finite rank, one has $\hat{N}=N$. We remark that for two modules, $N_{1}, N_{2}$, there is a natural map $\hat{N}_{1} \otimes_{\mathbb{L}} \hat{N}_{2} \rightarrow\left(N_{1} \otimes_{\mathbb{L}} N_{2}\right)$, which is an isomorphism when both modules are finitely generated and free.

We fix a $\mathbb{L}$-projective group object $G$, and write $S=L^{*, *}(G)$. The module $\hat{S}$ is in fact a ring, due to the Hopf-algebra structure on $S$. If $G$ acts on an object $Y$ 
of $s \mathbf{S h}_{\tau}(\mathbf{C})$ on the left, then the action map $G \times Y \rightarrow Y$, along with compatibility diagrams, imbues $L^{*, *}(Y)$ with an $S$-comodule structure. Alternatively, the dual of $L^{*, *}(Y)$ is a module over $\hat{S}$.

Theorem 1.7 Let $G$ be an $\mathbb{L}$-projective group object, and let $Y \in s \mathbf{S h}_{\tau}(\mathbf{C})$ be a simplicial sheaf on which $G$ acts on the left. Suppose $N=L^{*, *}(Y)$ is a graded free $\mathbb{L}$-module and write $S$ for the Hopf algebra $L^{*, *}(G)$. There is a spectral sequence

$$
\mathrm{E}_{2}^{p, q}=\operatorname{Ext}_{\widehat{S}}^{p, q, *}(\widehat{N}, \mathbb{L})
$$

which is functorial in $Y, G$ and $L^{*, *}$.

Moreover, the product structure on this spectral sequence is given on the $\mathrm{E}_{2}$-page by the natural product structure on $\operatorname{Ext}_{\widehat{S}}^{*}(\widehat{N}, \mathbb{L})$, for which see Proposition A.3.

If the cohomology theory $L^{*, *}$ is bounded below on the set $\left\{B(G, Y)_{s}\right\}_{s=0}^{\infty}$, then the spectral sequence in question is strongly convergent:

$$
\mathrm{E}_{2}^{p, q}=\operatorname{Ext}_{\widehat{S}}^{p, q, *}(\widehat{N}, \mathbb{L}) \Longrightarrow L^{p+q, *}(B(G, Y)) .
$$

Proof Since $L^{*, *}(G)$ is a free $\mathbb{L}$-module, and $G$ is $\mathbb{L}$-projective, a Künneth isomorphism holds:

$$
L^{*, *}\left(B(G, Y)_{p}\right)=L^{*, *}(G)^{\otimes p} \otimes_{\mathbb{L}} L^{*, *}(Y)=S^{\otimes p} \otimes_{\mathbb{L}} N .
$$

We now consider the bar complex of $\hat{N}$ as a $\widehat{S}$-module, relative to $\mathbb{L}$. It is a simplicial $S$-module the $p$-simplices of which are

$$
\overbrace{\hat{S} \otimes_{\mathbb{L}} \hat{S} \otimes_{\mathbb{L}} \ldots \otimes_{\mathbb{L}} \hat{S}}^{p+1} \otimes_{\mathbb{L}} \hat{N} ;
$$

see Weibel [23, chapter 8]. Application of $\operatorname{Hom}_{\widehat{S}}(\cdot, \mathbb{L})$ to this complex yields a cosimplicial $\mathbb{L}$-module whose $p$-simplices are

$$
\operatorname{Hom}_{\widehat{S}}\left(\widehat{S}^{\otimes p+1} \otimes_{\mathbb{L}} \hat{N}, \mathbb{L}\right) \cong S^{\otimes p} \otimes_{\mathbb{L}} N,
$$

where the natural isomorphism indicated is deduced by elementary ring theory. One can verify by element-level calculations that the structure maps in this cosimplicial $\mathbb{L}$-module are precisely those of the cosimplicial $\mathbb{L}$-module $L^{*, *}(B(G, Y) \bullet)$. Since the bar complex is in this case a free resolution of $\widehat{N}$ as an $\widehat{S}$-module, it follows that the $\mathrm{E}_{2}$-page of the spectral sequence is precisely $\operatorname{Ext}_{\widehat{S}}^{p, q}(\widehat{N}, \mathbb{L})$ as promised.

For the most part now we devote ourselves to computing this spectral sequence or its abutment. Our first result towards this end is the following, which will allow us to compute $L^{*, *}(B(G, Y))$ by decomposition of $Y$. 
Proposition 1.8 Let $G$ be a group object in $s \mathbf{S h}_{\tau}(\mathbf{C})$. Let $I$ be a small category and let $F: I \rightarrow s \mathbf{S h}_{\tau}(\mathbf{C})$ be a diagram in which all objects $F(i)$ are equipped with a $G$-action and such that the morphisms $F(i \rightarrow j)$ are $G$-equivariant. Then there is a weak equivalence hocolim $|B(G, F(I)) \bullet| \simeq|B(G, \operatorname{hocolim} F(I)) \bullet|$.

The proof relies on commuting homotopy colimits.

\subsection{The case of a free action}

A set-theoretically free action of a group $G$ on a set $X$ is an action for which the stabilizer of every element $x \in X$ is the trivial subgroup $\{e\} \subset G$. This may be rephrased as the condition that the map $G \times X \rightarrow X \times X$ given by $(g, x) \mapsto(g x, x)$ is an injection of sets. This motivates the following definition.

Definition 2 If $G$ is a group object in $s \mathbf{S h}_{\tau}(\mathbf{C})$, acting on an object $Y$ of $s \mathbf{S h}_{\tau}(\mathbf{C})$, such that the map $G \times Y \rightarrow Y \times Y$ is injective, then we say the action is free.

When the action of $G$ on $Y$ is free, one might hope that the simplicial Borel construction and the homotopy type of the quotient agree. One must be careful not confuse the quotient in $s \mathbf{S h}_{\tau}(\mathbf{C})$ with other notions of quotient that may exist internally to $\mathbf{C}$.

We are trying to imitate the following fact, true in the context of simplicial sets.

Proposition 1.9 Let $X$ be a simplicial set, and $G$ a simplicial group, acting freely on $X$. Then there is a map $B(G, X) \rightarrow X / G$ which is a weak equivalence. This map is natural, in that $(G, X) \rightarrow\left(G^{\prime}, X^{\prime}\right)$ induces a diagram

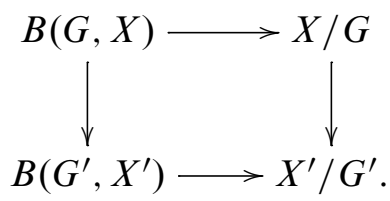

See [11, Chapter 8] for the proof.

We specify that if $G$ is a group object in $s \mathbf{S h}_{\tau}(\mathbf{C})$ and if $X$ is an object in $s \mathbf{S h}_{\tau}(\mathbf{C})$ on which $G$ acts, then the notation $X / G$ is to mean the 'orbit sheaf' in $s \mathbf{S h}_{\tau}(\mathbf{C})$. This is the quotient sheaf associated to the presheaf

$$
U \mapsto \operatorname{coeq}(G(U) \times X(U) \rightrightarrows X(U)),
$$

$v i z$ the presheaf of ordinary group quotients. Even when $X, G$ are represented by objects of $\mathbf{C}$, for instance if they are sheaves represented by schemes, we shall never write $X / G$ for any other quotient than this sheaf-theoretic quotient. 
Definition 3 Let $Y \rightarrow X$ be a surjective map in $s \mathbf{S h}_{\tau}(\mathbf{C})$ and let $G$ be a group object in $s \mathbf{S h}_{\tau}(\mathbf{C})$ such that there is an action of $G$ on $Y$, denoted $\alpha: G \times Y \rightarrow Y$, and that $G$ acts trivially on the object $X$, and that the map $Y \rightarrow X$ is $G$ equivariant. This is equivalent to saying the outer square in the diagram below is commutative:

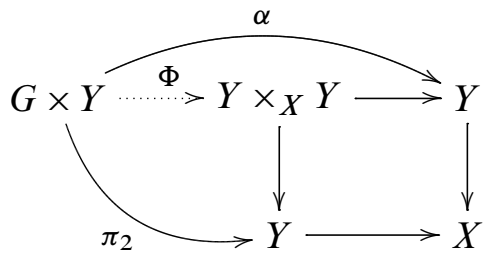

Under these assumptions there exists a natural map $\Phi: G \times Y \rightarrow Y \times_{X} Y$. If $\Phi$ is an isomorphism, then we say that $Y \rightarrow X$ is a principal $G$-bundle. We abbreviate the data of a $G$-bundle to $Y \rightarrow X$, where the $G$ action on $Y$ is understood.

A map of $G$-bundles $Y \rightarrow X$ to $Y^{\prime} \rightarrow X^{\prime}$ is a pair of $G$-equivariant maps $Y \rightarrow Y^{\prime}$ and $X \rightarrow X^{\prime}$ making the obvious square commute. Note that the $G$-equivariance of $X \rightarrow X^{\prime}$ is always trivially satisfied.

There are two maps $G \times Y \rightarrow Y$, both $\alpha$, the action map, and $\pi_{2}$, projection on the second factor. In tandem, they yield a map $\alpha \times \pi_{2}: G \times Y \rightarrow Y \times Y$, which is commonly denoted $\Psi$. When we refer to a map $G \times Y \rightarrow Y \times Y$ without expressly naming one, it is to be understood that $\Psi$ is intended.

Proposition 1.10 If $Y \rightarrow X$ is a principal $G$-bundle, then the action of $G$ on $Y$ is free.

The proof is immediate from the case of sets, since monomorphisms of sheaves may be detected objectwise.

Proposition 1.11 Let $X$ be an object in $s \mathbf{S h}_{\tau}(\mathbf{C})$, let $G$ be a group object in $s \mathbf{S h}_{\tau}(\mathbf{C})$, and suppose the action of $G$ on $X$ is free. Then there is an injective weak equivalence $|B(G, X)| \simeq X / G$ in $s \mathbf{S} \mathbf{h}_{\tau}(\mathbf{C})$. This weak equivalence is natural in both $G$ and $X$.

Proof For any $U \in \mathbf{C}$, we have $B(G, X)(U)=B(G(U), X(U))$, and so it follows

$$
B(G, X)(U) \simeq X(U) / G(U),
$$

and $X(U) / G(U)=(X / G)^{\text {pre }}(U)$, the latter being the presheaf quotient. It follows that $|B(G, X)| \simeq(X / G)^{\text {pre }}$ in the model category of presheaves. By [10], we know that $(X / G)^{\mathrm{pre}} \simeq X / G$, the latter being the quotient sheaf for the $\tau$-topology. 
One source of bundles is: If $G \in s \mathbf{S h}_{\tau}(\mathbf{C})$ is a group object, and $G$ acts on $X \in s \mathbf{S h}_{\tau}(\mathbf{C})$, and if $X$ has a point $x_{0}: \mathrm{pt} \rightarrow X$, then there is a map $G \times \mathrm{pt} \rightarrow G \times X \rightarrow X$. In the composition, we denote this by $f: G \rightarrow X$. Let $H$ be the pullback as given by

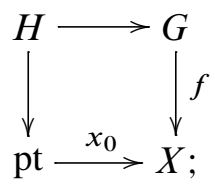

then $H$ is called the stabilizer of $x_{0}$ in $G$. It is easily seen that $H$ is a subgroup object of $G$. As a result, there is a group action (on the right) of $H$ on $G$ given by multiplication $G \times H \rightarrow G$.

Proposition 1.12 With notation as above, if the map $G \rightarrow X$ is surjective then $G \rightarrow X$ is a principal $H$-bundle.

The proof proceeds by checking the conditions on sections; there they are elementary. The surjectivity of $G \rightarrow X$ is a transitivity condition in the category of sheaves.

\subsection{Quotients of representables}

Recall that there is a Yoneda embedding $h: \mathbf{C} \rightarrow s \mathbf{P r e}(\mathbf{C})$. We say that a simplicial sheaf is representable if it is in the essential image of this embedding. We do not demand that all objects of $\mathbf{C}$ represent sheaves. We shall generally denote an object of $\mathbf{C}$ and the presheaf or sheaf it represents by the same letter.

The following lemma is elementary, and may be proved by considering points.

Lemma 1.13 Let $\pi: A \rightarrow B$ be a map in $s \mathbf{S h}_{\tau}(\mathbf{C})$. Then there are two maps $A \times{ }_{B} A \rightarrow A$, being the projection on the first and second factor respectively. Suppose $A \rightarrow B$ is a simplicial sheaf epimorphism, then

$$
A \times B=A \longrightarrow B
$$

is a coequalizer diagram of simplicial sheaves.

Proposition 1.14 Let $G$ be a group object in $s \mathbf{S h}_{\tau}(\mathbf{C})$, which acts on the object $Y$, and trivially on the object $X$. Let $\pi: Y \rightarrow X$ be a $G$-equivariant map, making $Y$ a principal $G$-bundle over $X$. Then there is an isomorphism $Y / G \cong X$, which is natural in $G, Y$ and $X$. 
Proof Using Lemma 1.13, we see that in the diagram

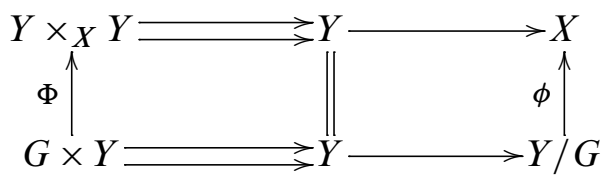

both sequences are coequalizer sequences. By assumption $\Phi: G \times Y \rightarrow Y \times_{X} Y$ is an isomorphism. By categorical uniqueness of coequalizers, the map $\phi: Y / G \rightarrow X$ is an isomorphism. The asserted naturality follows immediately by considering diagrams of coequalizer sequences, and is routine.

Corollary 1.14.1 Let $G$ be a group object in $s \mathbf{S h}_{\tau}(\mathbf{C})$, which acts on the object $Y$, and trivially on the object $X$. Let $\pi: Y \rightarrow X$ be a principal $G$-bundle. Then there is a weak equivalence $B(G, Y) \simeq X$, which is natural in $G, Y$ and $X$.

Proof This follows from the weak equivalence $B(G, Y) \simeq Y / G$.

Suppose that if $X, Y$ and $G$ are representable objects in $s \mathbf{S h}_{\tau}(\mathbf{C})$. If we consider them as objects in $\mathbf{C}$, then of all the hypotheses of the two results above, only the statement that $\pi: Y \rightarrow X$ is a surjective map of sheaves cannot be verified in $\mathbf{C}$ without reference to the topology. To verify that a map $\pi: Y \rightarrow X$ is a surjective map of sheaves, we must find a family of maps $\left\{f_{j}: U_{j} \rightarrow X\right\}$ which are covering for the topology $\tau$, and which have the property that there are sections $s_{j}: U_{j} \rightarrow Y$ satisfying $\pi \circ s_{j}=f_{j}$, in this case we say $\pi$ admits $\tau$-local sections.

Combining the above with Theorem 1.7 gives the following.

Theorem 1.15 Let $G$ be an $\mathbb{L}$-projective representable group object, and abbreviate the cohomology as $S=L^{*, *}(G)$. Suppose we have a map of representable objects, $\pi: Y \rightarrow X$, which admits $\tau$-local sections, and which is a principal $G-b u n d l e . ~ S u p p o s e$ that $G$ and $Y$ belong to a subcategory $\mathbf{U}$ of $s \mathbf{S h}_{\tau}(\mathbf{C})$ which is closed under formation of products and such that $L^{*, *}$ is $\mathbf{U}$-bounded below. Suppose further that the cohomology of $Y$, denoted $N=L^{*, *}(Y)$, is free and finitely generated as an $\mathbb{L}$-module. There exists a strongly convergent spectral sequence of algebras

$$
\mathrm{E}_{2}^{p, q}=\operatorname{Ext}_{\widehat{S}}^{p, q}(\hat{N}, \mathbb{L}) \Longrightarrow L^{p+q, *}(X ; R),
$$

and this spectral sequence is functorial in $(G, Y)$. The differentials take the form $d_{r}: \mathrm{E}_{r}^{p, q} \rightarrow \mathrm{E}_{r}^{p+r, q-r+1}$.

The functoriality is exactly the usual functoriality of Ext. 


\section{The equivariant cohomology of Stiefel varieties}

The theme of this section is the application the tools of the previous section to the case of actions of $\mathbb{G}_{m}$ on Stiefel varieties. The signal results are the almost complete calculation (by which we mean the identification of the $\mathrm{E}_{\infty}$-page of a convergent spectral sequence) of the motivic cohomology of $B\left(\mathbb{G}_{m}, \mathrm{GL}_{n}\right)$ with a general $\mathbb{G}_{m^{-}}$ action in Proposition 2.15, and the partial calculation of the analogue with $\mathrm{GL}_{n}$ replaced by a more general Stiefel variety in Theorem 2.19 .

In classical topology, one might consider the fibration $X \rightarrow X \times_{G} E G \rightarrow B G$ and then employ a Serre spectral sequence to go from knowledge of $\mathrm{H}^{*}(X)$ and $\mathrm{H}^{*}(B G)$ to $\mathrm{H}^{*}\left(X \times_{G} E G\right)$. This is what we do in spirit, since if we take that fiber sequence and start to extend it to a Puppe sequence, we should arrive at $G \simeq \Omega B G \rightarrow X \rightarrow X \times{ }_{G} E G$, which is equivalent to the fiber sequence $G \rightarrow E G \times X \rightarrow E G \times{ }_{G} X \simeq B(G, X)$. As a consequence of our going this roundabout way, the spectral sequences we obtain have $E_{2}$-pages resembling the $E_{3}$-pages of the Serre spectral sequences for which they are substitutes.

\subsection{Generalities}

We calculate with simplicial sheaves in the $\mathbb{A}^{1}$-model structure on the category $s \mathbf{S h}_{N i s}(\mathbf{S m} / k)$. Motivic cohomology is employed, $U \mapsto \mathrm{H}^{*, *}(U ; R)$; for a full treatment of this theory see Mazza, Voevodsky and Weibel [13] and Voevodsky [22]. For any commutative ring $R$, there exists a bigraded cohomology theory $\mathrm{H}^{*, *}(\cdot ; R)$, the definition of which is functorial in $R$.

Throughout $k$ will denote a field, on which further restrictions shall be placed later. By $\mathbf{S m}_{k}$ we mean, as is customary, [14], the category of smooth, separated, finite type $k$-schemes; the objects of this category will be referred to simply as smooth schemes in the sequel. The topology is the Nisnevich topology, for which see Nisnevich [15] or [14].

We enumerate some of the properties of $\mathrm{H}^{*, *}$. In particular, one has the following.

Proposition 2.1 Let $U$ be a smooth scheme, then $\mathrm{H}^{p, q}(U ; R)=\mathrm{CH}^{q}(U, 2 q-p)_{R}$, where the latter is the $R$-valued higher Chow groups of Bloch [1].

In order to use our convergence results, we will need to know some boundedness result for motivic cohomology. The Beilinson-Soule vanishing conjecture is that for a smooth scheme $X$, one has $\mathrm{H}^{p, q}(X ; \mathbb{Z})=0$ when $p<0$. This is known only in certain contexts at present. 
The following result is folklore. It is proved by using the Bloch-Kato conjecture, which is now proved, to reduce the question for all fields to the problem with $\mathbb{Q}$ coefficients. With $\mathbb{Q}$-coefficients, the motivic spectral sequence converging to $K-$ theory is degenerate, and for finite fields or number fields the higher $K$-theory with $\mathbb{Q}$-coefficients is known.

Proposition 2.2 The Beilinson-Soule vanishing conjecture holds for the motivic cohomology $\mathrm{H}^{p, q}(\operatorname{Spec} F ; \mathbb{Z})$ when $F$ is a finite field or a number field.

For any commutative ring $R$, the ring $\mathrm{H}^{*, *}(\operatorname{Spec} k ; R)$ will be denoted $\mathbb{M}_{R}$ or $\mathbb{M}$ where $R$ is understood. We shall write $\mathbb{M}^{i, j}$ to denote $\mathrm{H}^{i, j}(\operatorname{Spec} k ; R)$. Observe that $\mathbb{M}^{0,0}=R$. Note that for any $Y$, the ring $N=\mathrm{H}^{*, *}(Y ; R)$ is an $\mathbb{M}_{R}$-module. We shall write $\hat{N}$ to denote the dual, $\hat{N}=\operatorname{Hom}_{\mathbb{M}_{R}}\left(N, \mathbb{M}_{R}\right)$. In addition to the Beilinson-Soulé vanishing conjecture, the following vanishing obtains the following.

Proposition 2.3 For any $X \in \mathbf{S m}_{k}$, one has $\mathrm{H}^{p, q}(X ; R)=0$ if $q<0$, or if $2 q-p<0$. In the case where $X$ is equidimensional of dimension $d$, one has $\mathrm{H}^{p, q}(X ; R)=0$ when $p-d-q<0$.

In the following theorem, 'stably cellular' is taken in the sense of [4].

Theorem 2.4 Let $G$ be a smooth, stably cellular group scheme over $k$ such that $S=\mathrm{H}^{*, *}(G ; R)$ is a finitely generated graded free $\mathbb{M}_{R}$-module, generated by elements in nonnegative bidegree. Suppose $G$ acts on a smooth scheme $Y$ such that $N=\mathrm{H}^{*, *}(Y ; R)$ is also a finitely generated free $\mathbb{M}_{r}$-module, again generated by elements in nonnegative bidegree. Then there is a strongly convergent spectral sequence of algebras:

$$
\mathrm{E}_{2}^{p, q}=\operatorname{Ext}_{\widehat{S}}\left(\hat{N}, \mathbb{M}_{R}\right) \Longrightarrow \mathrm{H}^{*, *}(B(G, Y) ; R),
$$

which is functorial in $G, Y$ and $R$. The differentials act as $d_{r}: \mathrm{E}_{r}^{p, q} \rightarrow \mathrm{E}_{r}^{p+r, q-r+1}$.

Proof This is a special case of Theorem 1.7.

Corollary 2.4.1 We continue the hypotheses of the theorem, and add the following: if the action of $G$ on $Y$ is free and if $X$ is a scheme representing the Nisnevich quotient $Y / G$ then the spectral sequence above converges to the motivic cohomology, $\mathrm{H}^{*, *}(X ; R)$ :

$$
\mathrm{E}_{2}^{p, q}=\operatorname{Ext}_{\widehat{S}}\left(\widehat{N}, \mathbb{M}_{R}\right) \Longrightarrow \mathrm{H}^{*, *}(X ; R) .
$$


Proof A free action is one for which the map $G \times Y \rightarrow Y \times Y$ is a monomorphism. Since the Yoneda embedding preserves monomorphisms, an action which is free in the scheme-theoretic sense is free in the sheaf-theoretic sense. The corollary follows from the theorem in conjunction with Proposition 1.11.

Theorem 2.5 Let $G$ be a smooth group scheme, and $X$ a smooth scheme on which $G$ acts. Suppose $x_{0} \rightarrow X$ is a $k$-point of $X$ and $H$ is the stabilizer of $x_{0}$. Write $S=\mathrm{H}^{*, *}(H ; R)$, and $N=\mathrm{H}^{*, *}(G ; R)$. Suppose both $S$ and $N$ are finitely generated free graded $\mathbb{M}_{R}$-modules, generated by classes of nonnegative bidegree. Denote by $f$ the obvious map, $f: G \times x_{0} \rightarrow G \times X \rightarrow X$. If this map is Nisnevich-locally split, in that there is a Nisnevich cover $c: U \rightarrow X$ and a splitting map $s: U \rightarrow f^{-1}(U)$ satisfying $c^{-1}(f) \circ s=\mathrm{id}_{U}$, then there is a strongly convergent spectral sequence of algebras:

$$
\mathrm{E}_{2}^{p, q}=\operatorname{Ext}_{\widehat{S}}\left(\hat{N}, \mathbb{M}_{R}\right) \Longrightarrow \mathrm{H}^{*, *}(X ; R) .
$$

Proof Since the Yoneda embedding commutes with the formation of limits, $G \rightarrow X$ is a principal $H$-bundle in $s \mathbf{S h}_{N i s}(\mathbf{S m} / k)$. The result follows from Proposition 1.12 and Theorem 1.15.

The cohomology $\mathrm{H}^{*, *}(B(G, Y) ; R)$ is a variant of the Borel-equivariant cohomology with respect to $G$. There is another, more geometric, definition of equivariant higher Chow groups, defined in [5], which is denoted $\mathrm{CH}_{G}^{*}(X, *)_{R}$. In general, for a group scheme for which $\mathrm{H}_{\text {ét }}^{1}(\cdot, G) \not \mathrm{H}_{N i s}^{1}(\cdot, G)$, the two definitions are different; we can at least offer the following comparison result which applies to the cases considered in the sequel.

Recall that a group $G$ is special if every principal étale $G$-bundle is locally trivial in the Zariski (and a fortiori in the Nisnevich) topology. The group schemes $\mathrm{GL}_{n}$ and $\mathrm{SL}_{n}$ are special, as are products of special group schemes; see Grothendieck [7].

The following is a version of a result from Esnault, Kahn, Levine and Viehweg [6, Appendix A]. The vanishing conjecture is included in order to ensure convergence of the spectral sequences that appear.

Proposition 2.6 Let $G$ be a special group scheme. Let $X$ be a smooth scheme on which $G$ acts, and suppose the Beilinson-Soule vanishing conjecture is known to hold for all spaces of the form $G^{\times n}$ and $G^{\times n} \times X$, including the special cases where $n=0$. Then $\mathrm{H}^{p, q}(B(G, X) ; R) \cong \mathrm{CH}_{G}^{q}(X, 2 q-p)_{R}$. 
Proof One calculates $\mathrm{CH}_{G}^{i}(X, 2 i-n)_{R}$ by finding a representation $V \cong \mathbb{A}^{N}$ of $G$ such that the locus $Z \subset V$ where the action of $G$ on $V$ is not free is of very high codimension (codimension in excess of $2 i+2$ will be sufficient for our purposes), and such that $V \rightarrow V / G$ is Zariski-locally trivial. Write $Q=(X \times(V \backslash Z)) /$ ét $G$, where the quotient / ét is the algebraic space quotient. We know from [5, Proposition 23] that $Q$ is in fact a scheme. The quotient map $X \times(V \backslash Z) \rightarrow(X \times(V \backslash Z)) /$ ét $G$ is surjective as a map of étale sheaves, and it follows that $X \times(V \backslash Z) \rightarrow(X \times(V \backslash Z) /$ ét $G$ is an étalelocally trivial principal $G$-bundle. Since $G$ is special, it is in fact a Nisnevich-locally trivial principal $G$-bundle. In particular, $Q \simeq B(G, X \times(V \backslash Z))$ by Corollary 1.14.1. One defines $\mathrm{CH}_{G}^{i}(X, 2 i-n)_{R}=\mathrm{CH}^{i}(Q, 2 i-n)_{R}$.

For the groups $G$ satisfying the hypotheses above, one may ensure further that $Z$ is a union of linear subspaces of $V$, in particular $V \backslash Z$ is cellular, and has cohomology which is finitely generated and free over $\mathbb{M}$. Therefore $X \times(V \backslash Z)$ satisfies the Beilinson-Soulé vanishing conjecture if $X$ does.

Proposition 1.6 establishes convergent spectral sequences

$$
\begin{gathered}
N_{p}\left(\mathrm{H}^{q, *}(B(G, X) ; R)_{\bullet}\right) \Rightarrow \mathrm{H}^{p+q}(B(G, X)), \\
N_{p}\left(\mathrm{H}^{q, *}(B(G, X \times(V \backslash Z)) ; R)\right) \Rightarrow \mathrm{H}^{p+q}(Q ; R) .
\end{gathered}
$$

On the other hand, $\mathrm{H}^{q, *}\left(B(G, X)_{s} ; R\right)=\mathrm{H}^{q, *}\left(B(G, X \times(V \backslash Z))_{s} ; R\right)$ for $0 \leq s \leq 2 i+1$ by hypothesis on $V$, so that the two convergent spectral sequences agree on every page in the region $p+q \leq i$. Since both converge strongly, the result follows.

Examples where the two theories differ are abound once we drop the hypothesis that the group scheme be special. Suppose that we consider $G=\mu_{2} \cong \mathbb{Z} / 2$ acting on $\mathbb{G}_{m}$ by $x \mapsto-x$, all over Spec $\mathbb{C}$ for concreteness. Then the étale-sheaf quotient $\mathbb{G}_{m} / G$, which is also the geometric quotient, is $\mathbb{G}_{m}$. It follows that $\mathrm{CH}_{G}^{*}\left(\mathbb{G}_{m}, *\right) \cong \mathrm{CH}^{*}\left(\mathbb{G}_{m}, *\right)$, although the isomorphism is not via the projection map $\mathbb{G}_{m} \rightarrow \mathbb{G}_{m} / G$. If we consider our Borel equivariant motivic cohomology, however, we obtain a spectral sequence from Theorem 2.4:

$$
\mathrm{E}_{2}^{*, *}=\operatorname{Ext}_{\mathbb{M}[G]}(\mathbb{M} \oplus \mathbb{M} \sigma, \mathbb{M}) \Longrightarrow \mathrm{H}^{*, *}\left(B\left(G, \mathbb{G}_{m}\right) ; \mathbb{Z}\right) .
$$

Here $\sigma$ is the dual of the generator of $\widetilde{\mathrm{H}}^{*, *}\left(\mathbb{G}_{m} ; \mathbb{Z}\right)$, one has $|\sigma|=(1,1)$, and one verifies (see Proposition 2.10) that $G$ acts trivially on $\mathbb{M} \oplus \mathbb{M} \sigma$. The ring $\mathbb{M}[G]$ may be presented as $\mathbb{M} \oplus \mathbb{M} g$, with $g^{2}=1$, and $|g|=(0,0)$. The trigraded $\mathrm{E}_{2}$-page may be described as $(\mathbb{M} \oplus \mathbb{M} \hat{\sigma}) \otimes_{\mathbb{Z}} \mathrm{H}_{\text {group }}^{*}(G, \mathbb{Z})$, where the group cohomology lies in tridegrees $(*, 0,0)$ and $\hat{\sigma}$ lies in tridegree $(0,1,1)$. In particular, $\mathrm{E}_{2}^{2 i, 0,0}=\mathbb{Z} / 2$ whenever $i>0$, but there are no elements of weight 0 in $\mathrm{E}_{2}^{*, p}$ where $p>0$. The classes in $\mathrm{E}_{2}^{2 i, 0,0}$ for 
$i>0$ cannot therefore be the source or target of any differential, and therefore persist to the $\mathrm{E}_{\infty}$-page. Since the spectral sequence converges, $\mathrm{H}^{2 i, 0}\left(B\left(G, \mathbb{G}_{m}\right) ; \mathbb{Z}\right)=\mathbb{Z} / 2$ for $i \geq 0$, and $B\left(G, \mathbb{G}_{m}\right)$ does not have the motivic cohomology of a smooth scheme.

\subsection{The ordinary motivic cohomology of Stiefel varieties}

Let $i$ and $n$ be positive integers satisfying $i \leq n$. We define a Stiefel variety $V_{i}\left(\mathbb{A}^{n}\right)$ to be the variety of $n \times i$-matrices having rank $i$. There are two special cases: $V_{1}\left(\mathbb{A}^{n}\right)=\mathbb{A}^{n} \backslash 0$ and $V_{n}\left(\mathbb{A}^{n}\right)=\mathrm{GL}_{n}$. The Stiefel variety $V_{i}\left(\mathbb{A}^{n}\right)$ is an open subscheme of $\mathbb{A}^{i n}$.

The following result is due to Pushin [16]. We write $\{a\} \in \mathrm{H}^{1,1}(\operatorname{Spec} k ; \mathbb{Z})$ for the element corresponding to $a \in k^{*}$, and we also write $\{a\}$ for the image of this class in $\mathrm{H}^{1,1}(X ; R)$ under the natural map induced by $X \rightarrow \operatorname{Spec} k$ and $\mathbb{Z} \rightarrow R$.

Theorem 2.7 Over any field, the motivic cohomology $\mathrm{H}^{* * *}\left(\mathrm{GL}_{n} ; R\right)$ is the almost exterior algebra generated over $\mathbb{M}$ by classes $\rho_{1}, \ldots, \rho_{n}$ in bidegrees $\left|\rho_{i}\right|=(2 i-1, i)$ and subject to the relation

$$
\rho_{i}^{2}= \begin{cases}0 & \text { if } 2 i-1>n, \\ \{-1\} \rho_{2 i-1} & \text { otherwise, }\end{cases}
$$

and to the usual constraints of graded commutativity in the first grading and commutativity in the second.

One may deduce the following corollaries, due to the author [24].

Corollary 2.7.1 Over any field, the motivic cohomology $\mathrm{H}^{*, *}\left(V_{m}\left(\mathbb{A}^{n}\right) ; R\right)$ is the subalgebra of $\mathrm{H}^{*, *}\left(\mathrm{GL}_{n} ; R\right)$ generated by $\rho_{n-m+1}, \ldots, \rho_{n}$. The usual projection $\mathrm{GL}_{n} \rightarrow V_{m}\left(\mathbb{A}^{n}\right)$ induces this inclusion on cohomology.

Proposition 2.8 The usual inclusion $\mathrm{GL}_{n} \rightarrow \mathrm{GL}_{n+1}$ induces the quotient map

$$
\mathrm{H}^{*, *}\left(\mathrm{GL}_{n+1} ; R\right) \rightarrow \mathrm{H}^{*, *}\left(\mathrm{GL}_{n+1} ; R\right) /\left(\rho_{n+1}\right) \cong \mathrm{H}^{*, *}\left(\mathrm{GL}_{n} ; R\right) .
$$

Proposition 2.9 The group multiplication $\mu$ : $\mathrm{GL}_{n} \times \mathrm{GL}_{n} \rightarrow \mathrm{GL}_{n}$ induces a comultiplication $\mu^{*}: \mathrm{H}^{*, *}\left(\mathrm{GL}_{n} ; R\right) \rightarrow \mathrm{H}^{*, *}\left(\mathrm{GL}_{n} ; R\right) \otimes_{\mathbb{M}} \mathrm{H}^{*, *}\left(\mathrm{GL}_{n} ; R\right)$. This is an algebra map, and is fully determined by $\mu^{*}\left(\rho_{i}\right)=1 \otimes \rho_{i}+\rho_{i} \otimes 1$.

Proof This follows immediately from the bigrading on $\mathrm{H}^{*, *}\left(\mathrm{GL}_{n} ; R\right)$.

Corollary 2.9.1 The group-inversion map $i: \mathrm{GL}_{n} \rightarrow \mathrm{GL}_{n}$ induces a map $i$ * on cohomology that is determined by $i^{*}\left(\rho_{i}\right)=-\rho_{i}$. 
Proof Observe that the composite of the diagonal $\Delta: \mathrm{GL}_{n} \rightarrow \mathrm{GL}_{n} \times \mathrm{GL}_{n}$, inversion on the first factor $\iota \times \mathrm{id}$, and multiplication $\mu$ : $\mathrm{GL}_{n} \times \mathrm{GL}_{n} \rightarrow \mathrm{GL}_{n}$ gives the 0 -map on cohomology. The result follows.

Corollary 2.9.2 The action $\alpha: \mathbb{G}_{m} \times \mathrm{GL}_{n} \rightarrow \mathrm{GL}_{n}$ given by multiplication of a row or column by a scalar induces a coaction

$$
\alpha^{*}: \mathrm{H}^{*, *}\left(\mathrm{GL}_{n} ; R\right) \rightarrow \mathrm{H}^{*, *}\left(\mathbb{G}_{m} ; R\right) \otimes \mathrm{H}^{*, *}\left(\mathrm{GL}_{n} ; R\right),
$$

which is given by $\alpha^{*}\left(\rho_{1}\right)=\rho_{1} \otimes 1+1 \otimes \rho_{1}$, and $\alpha^{*}\left(\rho_{i}\right)=1 \otimes \rho_{i}$.

Proposition 2.10 There is a $\mu_{2}$ action on $\mathbb{A}^{n} \backslash\{0\}$ given by $\mathbf{v} \mapsto-\mathbf{v}$. The induced $\mu_{2}(k)$-action on $\mathrm{H}^{*, *}\left(\mathbb{A}^{n} \backslash\{0\} ; R\right)$ is trivial.

Proof The $\mu_{2}(k)$-action on the whole ring may be deduced from the $\mu_{2}(k)$ action on $\mathrm{H}^{2 n-1, n}\left(\mathbb{A}^{n} \backslash\{0\} ; R\right)=\mathrm{CH}^{n}\left(\mathbb{A}^{n} \backslash\{0\}, 1\right)_{R}$, since this group and $\mathrm{CH}^{0}\left(\mathbb{A}^{n} \backslash\{0\}, 0\right)_{R}$ serve to generate the ring. Since the $\mu_{2}(k)$-action extends to an action on $\mathbb{A}^{n} \simeq \mathrm{pt}$ fixing the origin, the result follows from functoriality of the localization sequence for higher Chow groups.

Proposition 2.11 There is a symmetric group, $\Sigma_{i}$, action on $V_{i}\left(\mathbb{A}^{n}\right)$, given by permuting the columns of matrices. This action is trivial on cohomology.

Proof By considering first the projection $\mathrm{GL}_{n} \rightarrow V_{i}\left(\mathbb{A}^{n}\right)$ and then the inclusion $\mathrm{GL}_{n} \rightarrow \mathrm{GL}_{n+1}$, one may assume $V_{i}\left(\mathbb{A}^{n}\right)$ is in fact $\mathrm{GL}_{n}$ for $n>3$. A transformation of the form $A \mapsto A \cdot E_{i j}(\lambda)$ where $E_{i j}(\lambda)$ is an elementary matrix induces the identity map $\mathrm{H}^{*, *}\left(\mathrm{GL}_{n} ; R\right) \rightarrow \mathrm{H}^{*, *}\left(\mathrm{GL}_{n} ; R\right)$, since there is an $\mathbb{A}^{1}$-homotopy of maps from multiplication by $E_{i j}(\lambda)$ to the identity. The transformation that interchanges two columns and changes the sign of one is a composite of such elementary transformations. By comparison with Proposition 2.10 we see that the change of sign also induces the identity on cohomology, so that it follows that all transpositions of columns induce the identity on cohomology. Since these serve to generate $\Sigma_{i}$, the result follows.

\subsection{Torus Actions on GL $_{n}$}

We compute some of examples of the Rothenberg-Steenrod spectral sequence for motivic cohomology, culminating in the case of $B\left(\mathbb{G}_{m}, \mathrm{GL}_{n}\right)$ for a $\mathbb{G}_{m}$-action on $\mathrm{GL}_{n}$ of the most general type. This is the content of Proposition 2.16. In general, the determination of the $E_{2}$-page of the spectral sequences is simply a calculation of Ext-groups, is not difficult, and relies on a reference to Propositions A.7 or A.8. The determination of the differentials is more involved, and relies on the nature of the objects being studied. 
Unless otherwise stated, we assume that all varieties are defined over a ground field $k$ such that the Beilinson-Soule vanishing conjecture is known to hold for $\mathrm{H}^{*, *}(\operatorname{Spec} k ; \mathbb{Z})$. We shall also assume that our ring of coefficients, $R$, is one in which 2 is invertible or else that $R$ is a ring of characteristic 2 and $k$ contains a square root of -1 . In practice, we shall use $R=\mathbb{Z}\left[\frac{1}{2}\right], R=\mathbb{Z} / p$ where $p$ is an odd prime, $R=\mathbb{Q}$ and $R=\mathbb{Z} / 2$, but the last only in the case where -1 is a square in $k$. Under either hypothesis, the element $\{-1\} \in \mathbb{M}_{R}^{1,1}$ vanishes, and the cohomology $\mathrm{H}^{*, *}\left(\mathrm{GL}_{n} ; R\right)$ is an exterior algebra.

This section makes extensive use of the trigraded nature of the motivic spectral sequences, and it is therefore convenient to have a notational convention for that grading. A homogeneous element $\alpha$ in the $j$-th page of a spectral sequence will be said to have tridegree $|\alpha|=(p, q, r)$ if it lies in homological degree $p$, motivic degree $q$ and weight $r$. This element corresponds to one that would classically be understood to lie in bidegree $(p, q)$, that is to say $p$ 'across' and $q$ 'up'. The differential $d_{j}$ invariably will take $\alpha$ in of tridegree $(p, q, r)$ to $d_{j} \alpha$ in tridegree $(p+j, q-j+1, r)$. We define the total Chow height of $\alpha$ to be tch $\alpha=2 r-p-q$, and we note that

$$
\operatorname{tch} d_{j} \alpha=\operatorname{tch} \alpha-1 \text {. }
$$

Since total Chow height is linear in each grading, we also have $\operatorname{tch} \alpha \beta=\operatorname{tch} \alpha+\operatorname{tch} \beta$. In general, (1) allows us to discount a great many potential differentials in the motivic spectral sequence, which is an advantage over the classical case, where we do not have the crutch of the weight filtration.

The first, and easiest, of the spectral sequences is the following.

Proposition 2.12 Let $\mathbb{G}_{m}$ act on $\mathbb{A}^{n} \backslash\{0\}$ via the diagonal action, that is to say the map given by $r \cdot\left(a_{1}, \ldots, a_{n}\right)=\left(r a_{1}, \ldots, r a_{n}\right)$.

Suppose $n>1$, then the $\mathrm{E}_{2}$-page of the associated spectral sequence in motivic cohomology is the $\mathbb{M}$-algebra $\mathbb{M}\left[\rho_{n}, \theta\right] /\left(\rho_{n}^{2}\right)$, with $\left|\rho_{n}\right|=(0,2 n-1, n)$ and $|\theta|=(1,1,1)$. There is a single nonvanishing differential of note, on the $n$-th page, satisfying $d_{n} \rho_{n}=\theta^{n}$. All other differentials are determined by this one.

Suppose $n=1$, then $\mathbb{G}_{m} \cong \mathbb{A}^{1} \backslash\{0\}$ and the spectral sequence is trivial.

Proof We consider only the case $n>1$, the case $n=1$ being trivial.

The group action of $\mathbb{G}_{m}$ on $\mathbb{A}^{n} \backslash\{0\}$ gives rise to the Zariski-locally trivial principal $\mathbb{G}_{m}$-bundle $\mathbb{A}^{n} \backslash\{0\} \rightarrow \mathbb{P}^{n-1}$. It follows that $\left|B\left(\mathbb{G}_{m}, \mathbb{A}^{n} \backslash\{0\}\right)\right| \simeq \mathbb{P}^{n-1}$, from Proposition 1.14. 
The calculation of the $E_{2}$-page of the spectral sequence is straightforward, by reference to Corollary 2.9.2 and then Proposition A.7. The $\mathrm{E}_{2}$-page is therefore $\mathbb{M}\left[\rho_{n}, \theta\right] /\left(\rho_{n}^{2}\right)$, with $\left|\rho_{n}\right|=(0,2 n-1, n)$ and $|\theta|=(1,1,1)$, and we need only determine the differentials supported by $\rho_{n}$ and $\theta$.

\begin{tabular}{|c|c|c|c|}
\hline$*$ & $*$ & $*$ & $*$ \\
\hline $\mathbb{M}^{0,0} \rho_{n}$ & $*$ & $d_{n}$ & $*$ \\
\hline$*$ & $*$ & $*$ & $\longrightarrow \mathbb{M}^{0,0} \theta^{n}$ \\
\hline$\vdots$ & $\vdots$ & $\vdots$ &.$\cdot$ \\
\hline * & $*$ & $\mathbb{M}^{0,0} \theta^{2}$ & 0 \\
\hline$*$ & $\mathbb{M}^{0,0} \theta$ & 0 & 0 \\
\hline $\mathbb{M}^{0,0}$ & 0 & 0 & 0 \\
\hline
\end{tabular}

Figure 1: The $E_{2}$-page of the spectral sequence converging to $\mathrm{H}^{*, *}\left(\mathbb{P}^{n-1} ; R\right)$. Only the elements on the $(i, i)$ diagonal lie in Chow height 0 . The lower triangle of zeroes is implied by Beilinson-Soulé vanishing.

For dimensional reasons, $\theta$ cannot support any nonzero differentials. The total Chow height of $\rho_{n}$ is 1 , and so if it is to support a differential, the image must be of total Chow height 0 . We have $\operatorname{tch} \theta=0$, and so $\operatorname{tch} \theta^{i}=0$. For any element of nonzero degree, $\mu$, in $\mathbb{M}$, one has $\operatorname{tch} \mu \theta^{i}>0$. If $\rho_{n}$ is to support a differential, it must take the form $d_{j} \rho_{n}=\ell \theta^{i}$, where $\ell \in \mathbb{M}^{0,0}$. Considering degrees we should have $(j, 2 n-j, n)=(i, i, i)$, so $i=j=n$. Since $\rho_{n}$ can support no other differential, the spectral sequence collapses by the $n+1-$ st page at the latest. The sequence converges to the motivic cohomology of $\mathbb{P}^{n-1}$, for which the corresponding group $\mathrm{H}^{2 n, n}\left(\mathbb{P}^{n-1} ; R\right)$ is 0 , it follows that $\ell$ is a unit. Without loss of generality, we can choose generators for the cohomology of $\mathbb{G}_{m}$ and of $\mathbb{A}^{n-1} \backslash\{0\}$ so that this unit is in fact 1 .

We write $A^{(n)}$ for the $n$-graded part of a graded object $A$. In an abuse of notation, if $I \subset A$ is an ideal, we will write $A^{(n)} / I$ for the quotient group $A^{(n)} /\left(I \cap A^{(n)}\right)$.

Proposition 2.13 Suppose $n \geq 1$. Let $T_{n}=\left(\mathbb{G}_{m}\right)^{n}$ act on $\mathbb{A}^{n} \backslash\{0\}$ via the action $\left(\lambda_{1}, \ldots, \lambda_{n}\right) \cdot\left(\mu_{1}, \ldots, \mu_{n}\right)=\left(\lambda_{1} \mu_{1}, \ldots, \lambda_{n} \mu_{n}\right)$. Then the $\mathrm{E}_{2}-$ page of the associated spectral sequence for motivic cohomology is the $\mathbb{M}$-algebra $\mathbb{M}\left[\rho_{n}, \theta_{1}, \ldots, \theta_{n}\right] /\left(\rho_{n}^{2}\right)$. There is a single nonvanishing differential of note, on the $n$-th page, satisfying $d_{n} \rho_{n}=\prod_{i=1}^{n} \theta_{i}$. All other differentials are determined by this one. 
Proof The proof proceeds by induction on $n$. The case $n=1$ has been handled above. The determination of the $\mathrm{E}_{2}$-page is straightforward, following from Corollary 2.9.2 and Proposition A.7. For dimensional reasons the elements $\theta_{i}$ cannot support nonzero differentials. The calculation reduces to the question of differentials supported by $\rho_{n}$. There is a map of group schemes $\Delta: \mathbb{G}_{m} \rightarrow T_{n}$, given by the diagonal, and a commutative diagram of group actions

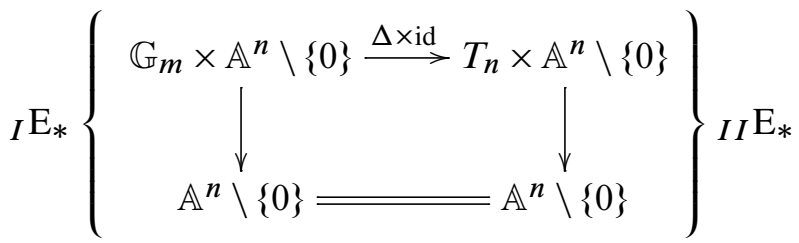

from which it follows that there is a map of spectral sequences, which we denote by $\Delta^{*}$. We refer to the spectral sequence for the $\mathbb{G}_{m}$-action as the first spectral sequence, that for the $T_{n}$-action as the second, and write ${ }_{I} \mathrm{E}_{*}^{*, *, *},{ }_{I I} \mathrm{E}_{*}^{*, *, *}$ to distinguish them. The map $\Delta^{*}$ goes from ${ }_{I I} \mathrm{E}_{*}$ to ${ }_{I} \mathrm{E}_{*}$. We have $\Delta^{*}\left(\rho_{n}\right)=\rho_{n}$ and $\Delta^{*}\left(\theta_{i}\right)=\theta$, the second conclusion following from the effect of the map $\Delta^{*}: \mathrm{H}^{*, *}\left(T_{n} ; \mathbb{Z}\right) \rightarrow \mathrm{H}^{*, *}\left(\mathbb{G}_{m} ; \mathbb{Z}\right)$ on cohomology along with some rudimentary homological algebra.

$\begin{array}{cccc}* & * & * & * \\ \rho_{n} & * & d_{n} & * \\ * & * & * & \mathbb{M}^{0,0}\left[\theta_{1}, \ldots, \theta_{n}\right]^{(n)} \\ \vdots & \vdots & \vdots & . \cdot \\ * & * & \mathbb{M}^{0,0}\left[\theta_{1}, \ldots, \theta_{n}\right]^{(2)} & 0 \\ * & \mathbb{M}^{0,0}\left[\theta_{1}, \ldots, \theta_{n}\right]^{(1)} & 0 & 0 \\ \mathbb{M}^{0,0} & 0 & 0 & 0\end{array}$

Figure 2: The $\mathrm{E}_{2}$-page of the spectral sequence ${ }_{I I} \mathrm{E}_{*}$. The notation $R^{(n)}$ denotes the $n$-th graded part of a graded ring $R$. The observations made in Figure 1 apply here as well.

In ${ }_{I I} \mathrm{E}_{2}$, an $m$-fold product of $\theta_{i}$ s has tridegree $(m, m, m)$, and a nontrivial multiple of this by a positively graded element of $\mathbb{M}$ has Chow height greater than 1 . It follows for similar reasons to those in Proposition 2.12 that $\rho_{n}$ cannot support any differential before $d_{n}\left(\rho_{n}\right)$. 
Since the term ${ }_{I I} \mathrm{E}_{n}^{n, n, n}$ is the group $\mathbb{M}^{0,0}\left[\theta_{1}, \ldots, \theta_{n}\right]^{(2 n, n)}$ of homogeneous polynomials, we must have

$$
d_{n} \rho_{n}=p\left(\theta_{1}, \ldots, \theta_{n}\right)
$$

for some homogeneous polynomial $p$ of degree $n$. We also know that

$$
\Delta^{*}\left(p\left(\theta_{1}, \ldots, \theta_{n}\right)\right)=p(\theta, \ldots, \theta)=\theta^{n} .
$$

Since there are no further differentials affecting this term of the spectral sequence, and no other terms of the spectral sequence converging to $\mathrm{H}^{2 n, n}\left(B\left(T_{n}, \mathbb{A}^{n} \backslash\{0\}\right) ; R\right)$, then

$$
\mathrm{H}^{2 n, n}\left(B\left(\mathbb{G}_{m}^{n}, \mathbb{A}^{n} \backslash\{0\}\right) ; R\right)=\frac{\mathbb{M}^{0,0}\left[\theta_{1}, \ldots, \theta_{n}\right]^{(n)}}{\left(p\left(\theta_{1}, \ldots, \theta_{n}\right)\right)},
$$

where $p\left(\theta_{1}, \ldots, \theta_{n}\right)$ is the homogeneous polynomial of degree $n$ which we wish to determine.

We decompose $\mathbb{A}^{n} \backslash\{0\}$ with the diagonal $T_{n}$ action into two open subschemes, $U_{1}=\mathbb{A}^{n-1} \backslash\{0\} \times \mathbb{A}^{1}$ and $U_{2}=\mathbb{A}^{n-1} \times \mathbb{A}^{1} \backslash\{0\}$. These open subschemes are $T_{n}$-invariant. By induction on $n$ and straightforward comparison, we have

$$
\begin{aligned}
\mathrm{H}^{*, *}\left(B\left(T_{n}, U_{1}\right) ; R\right) & =\frac{\mathbb{M}\left[\theta_{1}, \ldots, \theta_{n}\right]}{\left(\prod_{i=1}^{n-1} \theta_{i}\right)}, \\
\mathrm{H}^{*, *}\left(B\left(T_{n}, U_{2}\right) ; R\right) & =\frac{\mathbb{M}\left[\theta_{1}, \ldots, \theta_{n}\right]}{\left(\theta_{n}\right)} .
\end{aligned}
$$

By comparison with $B\left(T_{n}, U_{1}\right)$ and $B\left(T_{n}, U_{2}\right)$, we deduce that $p\left(\theta_{1}, \ldots, \theta_{n}\right)$ is a degree- $n$ polynomial which lies in the ideals $\left(\prod_{i=1}^{n-1} \theta_{i}\right)$ and $\left(\theta_{n}\right)$. The polynomial $p$ must therefore be $a \prod_{i=1}^{n} \theta_{i}$, where $a \in \mathbb{M}^{0,0}$. By reference to (2), we see that $a=1$.

We write $\sigma_{i}$ for the $i$-th elementary symmetric polynomial in $\mathbb{Z}\left[x_{1}, \ldots, x_{n}\right]$, and by extension if $\theta_{1}, \ldots, \theta_{n}$ are $n$ elements in any ring, we write $\sigma_{i}\left(\theta_{j}\right)$ to denote the $i$-th elementary symmetric polynomial in the $\theta_{j}$.

Proposition 2.14 Suppose $n \geq 1$. Let $T_{n}=\left(\mathbb{G}_{m}\right)^{n}$ act on $\mathrm{GL}_{n}$ via the action of left multiplication by diagonal matrices. Then the $\mathrm{E}_{2}$-page of the associated spectral sequence takes the form

$$
\mathrm{E}_{2}^{*, *}=\frac{\Lambda_{\mathbb{M}}\left(\rho_{2}, \ldots, \rho_{n}\right)\left[\theta_{1}, \ldots, \theta_{n}\right]}{\left(\sum_{i=1}^{n} \theta_{i}\right)}, \quad\left|\rho_{i}\right|=(0,2 n-1, n),\left|\theta_{i}\right|=(1,1,1),
$$

and the differentials are generated by $d_{i}\left(\rho_{i}\right)=\sigma_{i}\left(\theta_{i}\right)$, modulo the image of previous differentials, for $2 \leq i \leq n$. 
Note This $E_{2}$-page can be thought of as coming from a fictitious $E_{1}$-page

$$
\Lambda_{\mathbb{M}}\left(\rho_{1}, \ldots, \rho_{n}\right)\left[\theta_{1}, \ldots, \theta_{n}\right],
$$

with nonzero differential $d_{1}\left(\rho_{1}\right)=\sum \theta_{i}$. In classical algebraic topology this $\mathrm{E}_{2}-$ page is isomorphic to the $E_{3}$-page of the Serre spectral sequence of the fibration $T_{n} \rightarrow E T_{n} \times_{T_{n}} \mathrm{GL}_{n} \rightarrow B \mathrm{GL}_{n}$.

Proof The proof proceeds by induction on $n$. When $n=1, T_{1} \cong \mathbb{G}_{m} \cong \mathrm{GL}_{1}$, so there is nothing to prove.

In the case $n>1$, we know that the $\mathrm{E}_{2}$-page of the spectral sequence takes the anticipated form from Proposition A.8.

We fix canonical maps $\mathrm{GL}_{n-1} \rightarrow \mathrm{GL}_{n}$ and $T_{n-1} \rightarrow T_{n}$, being the standard inclusions, as in Proposition 2.8, The following diagram of group actions commutes:

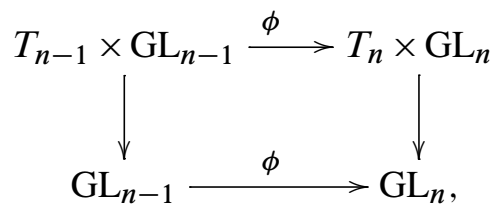

where we denote the morphisms of the group action by $\phi$, and use this to label the arrows in an abuse of notation. We obtain a comparison of spectral sequences, the pages of which we will denote by ${ }_{I I I} \mathrm{E}_{*}$ and ${ }_{I V} \mathrm{E}_{*}$. We have a map $\phi^{*}:{ }_{I V} \mathrm{E}_{*} \rightarrow{ }_{I I I} \mathrm{E}_{*}$.

By reference to Proposition A.8, we may fix presentations

$$
\begin{aligned}
I I I_{I} \mathrm{E}_{2}^{*, *} & =\frac{\Lambda_{\mathbb{M}}\left(\rho_{2}^{\prime}, \ldots, \rho_{n-1}^{\prime}\right)\left[\theta_{1}^{\prime}, \ldots, \theta_{n-1}^{\prime}\right]}{\left(\sum \theta_{i}^{\prime}\right)}, \\
I V \mathrm{E}_{2}^{*, *} & =\frac{\Lambda_{\mathbb{M}}\left(\rho_{2}, \ldots, \rho_{n}\right)\left[\theta_{1}, \ldots, \theta_{n}\right]}{\left(\sum \theta_{i}\right)} .
\end{aligned}
$$

We have $\phi^{*}\left(\rho_{i}\right)=\rho_{i}^{\prime}$, and $\phi^{*}\left(\theta_{i}\right)=\theta_{i}^{\prime}$ for $i<n$.

The usual argument by total Chow height prohibits all differentials supported by $\theta_{i}$, and restricts the possible differentials supported by $\rho_{i}$ to

$$
d_{i}: \mathbb{M}^{0,0} \rho_{i} \rightarrow{ }_{I V} \mathrm{E}_{i}^{i, i, i}
$$

We determine these $d_{i}$-differentials when $i<n$ by comparison with ${ }_{I I I} \mathrm{E}_{*}^{*, *}$.

Given a permutation $\alpha \in \Sigma_{n}$, the symmetric group on $n$ letters, there are maps $f_{\alpha}: \mathrm{GL}_{n} \rightarrow \mathrm{GL}_{n}$ and $g_{\alpha}: T_{n} \rightarrow T_{n}$ given by permuting the columns in the first case 


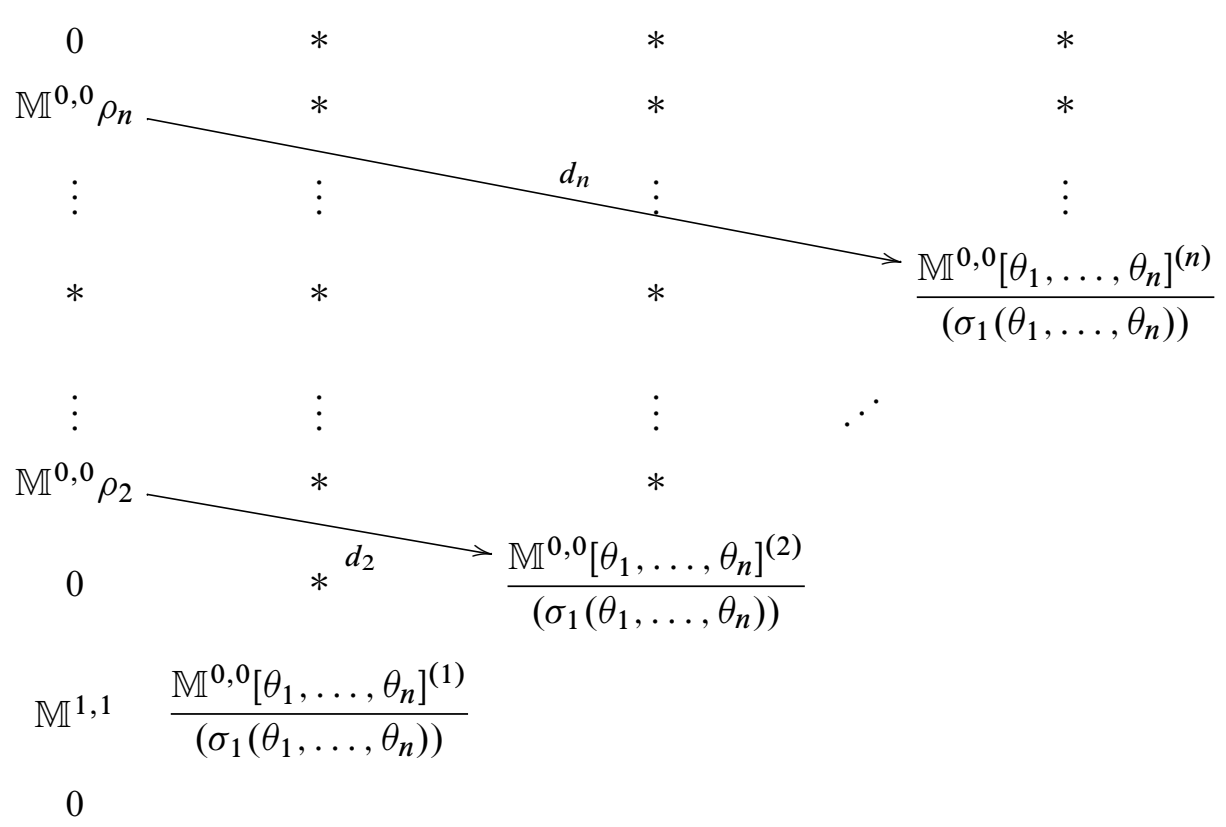

Figure 3: The $\mathrm{E}_{2}-$ page of the spectral sequence ${ }_{I V} \mathrm{E}_{*}$. In the first column, only the terms of Chow height 1 are shown. The $d_{n}$-differential is indicated in an abuse of notation, in reality, the target of this differential is a quotient of the illustrated group.

and permuting the multiplicands in the second. It is apparent that

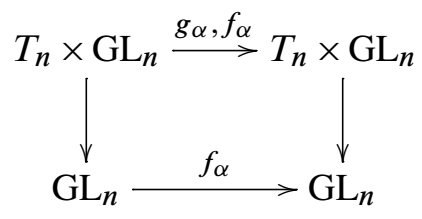

commutes, and as a result we obtain a $\Sigma_{n}$-action on the spectral sequence ${ }_{I V} \mathrm{E}_{*}^{*, *}$. The action of $g_{\alpha}^{*}$ on $\mathrm{H}^{*, *}\left(T_{n} ; R\right)$ is to permute the generators, whereas the action of $f_{\alpha}^{*}$ is trivial, by Proposition 2.11. In particular this implies that the action on the $\mathrm{E}_{2}$-page of the spectral sequence is to permute the classes $\theta_{i}$, but to fix those of the form $\rho_{j}$, so that $d_{i}\left(\rho_{i}\right) \in \mathrm{E}_{i}^{i, i, i}$ is represented by a homogeneous symmetric polynomial of degree $i$ in the $\theta_{i}$.

Suppose $i<n$, then by comparison and induction, we have

$$
\phi^{*} d_{i}\left(\rho_{i}\right)=d_{i} \rho_{i}^{\prime}=\left[\sigma_{i}\left(\theta_{1}^{\prime}, \ldots, \theta_{n-1}^{\prime}\right)\right],
$$


where $[\cdot]$ indicates the reduction of a class in $\mathbb{M}^{0,0}\left[\theta_{1}^{\prime}, \ldots, \theta_{n}^{\prime}\right]^{(i)}$ by the ideal generated by the images of prior differentials and $\sum \theta_{i}^{\prime}$. The map $\phi^{*}$ on $\mathrm{E}_{2}$-pages is given by evaluation at $\theta_{n}=\rho_{n}=0$. Elementary arguments now suffice to deduce that $p\left(\theta_{1}, \ldots, \theta_{n}\right)=\sigma_{i}\left(\theta_{1}, \ldots, \theta_{n}\right)$.

As for the case of $d_{n}\left(\rho_{n}\right)$, the above-stated comparisons tell us nothing. On the other hand, there is a diagram of group actions

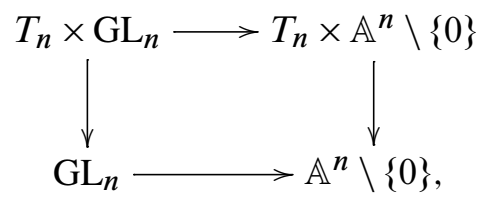

where the underlying map of $T_{n}$-spaces is simply projection on the first column, the effect of which on cohomology was computed in Corollary 2.7.1. It follows easily from comparison of spectral sequences with that of Proposition 2.13 that $d_{n}\left(\rho_{n}\right)=\sigma_{n}\left(\theta_{i}\right)$ in $I V \mathrm{E}_{*}^{*, *}$ as required.

Write $\operatorname{diag}\left(a_{1}, a_{2}, \ldots, a_{n}\right)$ for the $n \times n$ matrix having entries $a_{1}, a_{2}, \ldots, a_{n}$ on the main diagonal, and 0 elsewhere.

Let $\mathbf{w}=\left(w_{1}, \ldots, w_{n}\right) \in \mathbb{Z}^{n}$ be a set of $n$ weights. We shall distinguish these from the weight filtration of motivic cohomology by referring to the latter always in full. We consider the action of $\mathbb{G}_{m}$ on $\mathrm{GL}_{n}$ given on $k$-algebra-valued-points by

$$
z \cdot A=\operatorname{diag}\left(z^{w_{1}}, z^{w_{2}}, \ldots, z^{w_{n}}\right) A .
$$

Proposition 2.15 For the given action of $\mathbb{G}_{m}$ on $\mathrm{GL}_{n}$, there is a spectral sequence converging to the motivic cohomology of $B\left(\mathbb{G}_{m}, \mathrm{GL}_{n}\right)$. The $\mathrm{E}_{2}$-page is given as

$$
\begin{gathered}
\mathrm{E}_{2}^{p, q}=\operatorname{Ext}_{\widehat{\mathrm{H}}^{*, *}\left(\mathbb{G}_{m}\right)}^{p, q}\left(\hat{\mathrm{H}}^{*, *}\left(\mathrm{GL}_{n}, \mathbb{M}\right)\right)=\frac{\Lambda_{\mathbb{M}}\left(\rho_{2}, \ldots, \rho_{n}\right)[\theta]}{\left(\sum_{i=1}^{n} w_{i} \theta\right)}, \\
\left|\rho_{i}\right|=(0,2 i-1, i), \quad|\theta|=(1,1,1),
\end{gathered}
$$

with differentials $d_{i}\left(\rho_{i}\right)=\sigma_{i}(\mathbf{w}) \theta^{i}$. There are no differentials supported on elements of the form $\alpha \theta^{i}$ where $\alpha \in \mathbb{M}^{*, *}$. The indicated differentials are the only differentials supported on classes of the form $\alpha \rho_{i}$ where $\alpha \in \mathbb{M}^{0,0}$. Besides the nonzero differentials implied by the differentials described already and the product structure, if $d_{i}(s) \neq 0$ for some element on the $\mathrm{E}_{i}$-page, then $s$ must lie in the ideal on the $\mathrm{E}_{i}$-page generated by elements of the form $\mu \rho_{j}$ where $j<i$ and where $\sigma_{j}(\mathbf{w}) \mu=0$.

If $R$ is a field, then the indicated differentials and the product structure determine all the differentials. 
Proof For dimensional reasons, the classes $\theta^{i}$, and therefore the classes $\alpha \theta^{i}$ where $\alpha \in \mathbb{M}$, cannot support a nonzero differential.

There is a map of group actions

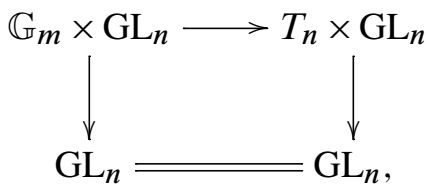

where the map of groups is $z \mapsto\left(z^{w_{1}}, z^{w_{2}}, \ldots, z^{w_{n}}\right)$. The map on cohomology induced by the group homomorphism is

$$
\begin{aligned}
\mathrm{H}^{*, *}\left(T_{n}\right) & \longrightarrow \mathrm{H}^{*, *}\left(\mathbb{G}_{m}\right), \\
\Lambda_{\mathbb{M}}\left(\tau_{1}, \ldots, \tau_{n}\right) & \longrightarrow \Lambda_{\mathbb{M}}(\tau), \\
\tau_{i} & \mapsto w_{i} \tau .
\end{aligned}
$$

This induces a map of $\mathrm{E}_{2}$-pages of spectral sequences

$$
\begin{aligned}
\operatorname{Ext}_{\widehat{\mathrm{H}} *, *\left(T_{n}\right)}\left(\hat{\mathrm{H}}^{*, *}\left(\mathrm{GL}_{n}\right), \mathbb{M}\right) & \rightarrow \operatorname{Ext}_{\widehat{\mathrm{H}}^{*, *}\left(\mathbb{G}_{m}\right)}\left(\hat{\mathrm{H}}^{*, *}\left(\mathrm{GL}_{n}, \mathbb{M}\right), \mathbb{M}\right), \\
\frac{\Lambda_{\mathbb{M}}\left(\rho_{2}, \ldots, \rho_{n}\right)\left[\theta_{1}, \ldots, \theta_{n}\right]}{\left(\sum \theta_{i}\right)} & \rightarrow \frac{\Lambda_{\mathbb{M}}\left(\rho_{2}, \ldots, \rho_{n}\right)[\theta]}{\left(\sum_{i=1}^{n} w_{i} \theta\right)}, \\
\rho_{i} \mapsto \rho_{i}, & \left|\rho_{i}\right|=(0,2 i-1, i), \\
\theta_{i} \mapsto w_{i} \theta, & |\theta|=(1,1,1) .
\end{aligned}
$$

By comparison with Proposition 2.14 the differentials $d_{i}\left(\rho_{i}\right)$ are already known. We have $d_{i}\left(\rho_{i}\right)=\sigma_{i}\left(w_{i}\right) \theta^{i}$. Any other nonzero differential on the $\mathrm{E}_{i}$-page must be supported on a term which is not in the image of the comparison map of $\mathrm{E}_{i}$-pages. Such terms are in the ideal generated by terms of the form $\mu \rho_{j}$ where $j<i$, and where $d_{j}\left(\mu \rho_{j}\right)=0$, so that $\mu \rho_{j}$ persists to the $\mathrm{E}_{i}$-page. Since $\rho_{j}$ per se cannot support any differentials beyond $d_{j}$ for dimensional reasons, it must be the case that $\mu \rho_{j}$ is not a multiple of $\rho_{j}$ on the $\mathrm{E}_{i}$-page, which means that $d_{j}\left(\rho_{j}\right)=\sigma_{j}(\mathbf{w}) \theta^{j} \neq 0$. This implies that $\sigma_{j}(\mathbf{w}) \neq 0$ in $\mathbb{M}^{0,0}$, but $\mu \rho_{j} \neq 0$ must persist to the $\mathrm{E}_{i}$-page, so $\mu \sigma_{j}(\mathbf{w})=0$. If $R=\mathbb{M}^{0,0}$ is a field, then $\sigma_{j}(\mathbf{w})$ is a unit in $\mathbb{M}^{0,0}$ so this cannot happen.

Corollary 2.15.1 Let $k$ be any field. Let $R$ be a field, and if $R$ is of characteristic 2 , then assume -1 is a square in $k$. Then $\mathrm{H}^{*, *}\left(\mathrm{PGL}_{n} ; R\right)$ admits the following presentation as an $\mathbb{M}_{R}$-algebra:

$$
\mathrm{H}^{*, *}\left(\mathrm{PGL}_{n} ; R\right) \cong \frac{\Lambda_{\mathbb{M}}\left(\rho_{1}, \ldots, \rho_{n}\right)[\theta]}{I}, \quad\left|\rho_{i}\right|=(2 i-1, i), \quad|\theta|=(2,1) .
$$


Here $I$ is generated by $\left(\rho_{i}, \theta^{i}\right)$, where $i$ is the least integer such that $\left(\begin{array}{c}n \\ i\end{array}\right) \neq 0$ in $R$. If further $R \cong \mathbb{Z} / p$, where $p$ is an odd prime, and $k$ is of characteristic different from $p$, then the action of the reduced power operations on $\mathrm{H}^{*}, *\left(\mathrm{PGL}_{n} ; \mathbb{Z} / p\right)$ is determined by $P^{i}\left(\rho_{j}\right)=\left(\begin{array}{c}j-i \\ i\end{array}\right) \rho_{i p+j-i}$ for $i p+j-i \leq n$ and $P^{i}(\theta)=\theta^{p}$ and the Cartan formula of Voevodsky [21].

Proof If $k$ is a field such that the Beilinson-Soule vanishing conjecture is known to hold for Spec $k$, then this is a corollary of Proposition 2.15 in the case where $\mathbf{w}=(1,1, \ldots, 1)$. In general, $\mathrm{PGL}_{n}$ gives rise to a compact object, $\Sigma^{\infty}\left(\mathrm{PGL}_{n}\right)_{+}$ of the stable homotopy category, [4]. Since PGL $\mathrm{PG}_{n} \simeq B\left(\mathbb{G}_{m}, \mathrm{GL}_{n}\right)$, and since $\mathbb{G}_{m}$ and $\mathrm{GL}_{n}$ are both stably cellular, it follows from the same place that $\mathrm{PGL}_{n}$ is also stably cellular.

Let $E$ denote either the prime-field of $k$ or the result of adjoining $\sqrt{-1}$ to the prime field if $R$ is of characteristic 2. There is a convergent Künneth spectral sequence

$$
\operatorname{Tor}^{\mathrm{H}^{*, *}(\operatorname{Spec} E ; R)}\left(\mathrm{H}^{*, *}\left(\mathrm{PGL}_{n, E} ; R\right), \mathbb{M}_{R}\right) \Longrightarrow \mathrm{H}^{*, *}\left(\mathrm{PGL}_{n, k} ; R\right) \text {. }
$$

Since $\mathrm{H}^{*, *}\left(\mathrm{PGL}_{n, E} ; R\right)$ is free as an $\mathrm{H}^{*, *}(\operatorname{Spec} E ; R)$-module, the spectral sequence is degenerate and the result follows.

The results regarding the reduced power operations follow immediately from the comparison $\mathrm{GL}_{n} \rightarrow \mathrm{PGL}_{n}$ and the analogous results for $\mathrm{H}^{*, *}\left(\mathrm{GL}_{n} ; R\right)$ in [24]. We note further that the Bockstein homomorphism

$$
\beta: \mathrm{H}^{*, *}\left(\mathrm{PGL}_{n} ; \mathbb{Z} / p\right) \rightarrow \mathrm{H}^{*+1, *}\left(\mathrm{PGL}_{n} ; \mathbb{Z} / p\right)
$$

vanishes on the classes $\theta^{i}$ for dimensional reasons, and that $\theta^{i}$, being a class in $\mathrm{H}^{*, *}\left(\mathrm{PGL}_{n} ; \mathbb{Z} / p\right)$ which is the reduction of a class in $\mathrm{H}^{*, *}\left(\mathrm{PGL}_{n} ; \mathbb{Z}\left[\frac{1}{2}\right]\right)$ cannot be the image of the Bockstein map. It follows that $\beta\left(\rho_{i}\right)=0$ for all $i$, and so it follows from the Cartan formulas that the action of the Steenrod algebra $\mathcal{A}_{p}$ on $\mathrm{H}^{*, *}\left(\mathrm{PGL}_{n} ; \mathbb{Z} / p\right)$ is fully determined.

In the case $p=2$, the argument above for deducing that $\beta\left(\rho_{i}\right)=0$ fails, because we have not calculated $\mathrm{H}^{*, *}\left(\mathrm{PGL}_{n} ; \mathbb{Z}\right)$. It is necessary only to know $\mathrm{CH}^{*}\left(\mathrm{PGL}_{n}\right)$, however, and this may be calculated using classical techniques as laid out in [7]. One again deduces that the classes $\theta^{i} \in \mathrm{H}^{2 i, i}\left(\mathrm{PGL}_{n} ; \mathbb{Z} / 2\right)$ are reductions of classes in $\mathrm{H}^{2 i, i}\left(\mathrm{PGL}_{n} ; \mathbb{Z}\right)$, which is all that is necessary to rule out a nonzero Bockstein map $\beta\left(\rho_{i}\right)$. The result of Corollary 2.15.1 therefore holds for $\mathbb{Z} / 2$ coefficients as well.

Our final result on the equivariant cohomology of $\mathrm{GL}_{n}$ deals with the equivariant cohomology for the following $\mathbb{G}_{m}$ action on the left and on the right. If $\mathbf{u}, \mathbf{v} \in \mathbb{Z}^{n}$ 
are two $n$-tuples of integers, one defines an action of $\mathbb{G}_{m}$ on $\mathrm{GL}_{n}$ as (on $k$-algebravalued-points)

$$
z \cdot A=\operatorname{diag}\left(z^{u_{1}}, z^{u_{2}}, \ldots, z^{u_{n}}\right) A \operatorname{diag}\left(z^{-v_{1}}, z^{-v_{2}}, \ldots, z^{-v_{n}}\right) .
$$

Proposition 2.16 Assume in this proposition that 2 is invertible in $R$.

For the $\mathbb{G}_{m}$ action given above, the equivariant cohomology spectral sequence has $\mathrm{E}_{2}$-page

$$
\mathrm{E}_{2}^{*, *}=\operatorname{Ext}_{\hat{\mathrm{H}}^{*, *}\left(\mathbb{G}_{m} ; R\right)}\left(\hat{\mathrm{H}}^{*, *}\left(\mathrm{GL}_{n} ; R\right), \mathbb{M}\right),
$$

which is an extension of $\Lambda_{\mathbb{M}}\left(\rho_{1}, \ldots, \rho_{n}\right)[\theta]$-modules

$$
\begin{aligned}
0 \rightarrow \frac{\Lambda_{\mathbb{M}}\left(\rho_{2}, \ldots, \rho_{n}\right)[\theta]}{\left(\left[\sum u_{i}-\sum v_{i}\right] \theta\right)} \rightarrow \operatorname{Ext}_{\widehat{\mathrm{H}}^{*, *}\left(\mathbb{G}_{m} ; R\right)}\left(\hat{\mathrm{H}}^{*, *}\left(\mathrm{GL}_{n} ; R\right), \mathbb{M}\right) \\
\quad \longrightarrow \operatorname{Ann}_{\Lambda_{\mathbb{M}}\left(\rho_{2}, \ldots, \rho_{n}\right)[\theta]}\left(\left[\sum u_{i}-\sum v_{i}\right] \theta\right) \rightarrow 0
\end{aligned}
$$

If $\sum u_{i}-\sum v_{i}=0$, then the $\mathrm{E}_{2}$-page can be written as

$$
\frac{\Lambda_{\mathbb{M}}\left(\rho_{1}, \ldots, \rho_{n}\right)[\theta]}{\left(\left[\sum u_{i}-\sum v_{i}\right] \theta\right)}=\operatorname{Ext}_{\widehat{\mathrm{H}}^{*, *}\left(\mathbb{G}_{m} ; R\right)}\left(\hat{\mathrm{H}}^{*, *}\left(\mathrm{GL}_{n} ; R\right) \mathbb{M}\right) .
$$

In this spectral sequence, there are differentials

$$
d_{i}\left(\rho_{i}\right)=\left[\sigma_{i}(\mathbf{u})-\sigma_{i}(\mathbf{v})\right] \theta^{i}\left(\bmod \sigma_{1}(\mathbf{u})-\sigma_{1}(\mathbf{v}), \ldots, \sigma_{i-1}(\mathbf{u})-\sigma_{i-1}(\mathbf{v})\right),
$$

where $i$ is any integer between 1 and $n$.

These are the only differentials supported on classes of the form $a \rho_{i}$ where $a \in \mathbb{M}^{0,0}$. There are no nonzero differentials supported on $\theta$. Besides the nonzero differentials implied by the differentials described already and the product structure, if $d_{i}(s) \neq 0$ for some element on the $\mathrm{E}_{i}$-page, then $s$ must lie in the ideal of the $\mathrm{E}_{i}$-page generated by elements of the form $\mu \rho_{j}$ where $j<i$ and where $\mu\left(\sigma_{j}(\mathbf{u})-\sigma_{j}(\mathbf{v})\right)=0$, that is to say that $\mu \rho_{j}$ is in the kernel of the $d_{j}$-differentials because of the torsion of $\mu$.

If $R$ is a field there are no further differentials other than those specified above and determined by the product structure.

The description of the $E_{2}$-page is understood as arising from a hypothetical $E_{1}-$ page that involves only the generators $\rho_{1}, \ldots, \rho_{n}$ and $\theta$, with a $d_{1}$-differential $d_{1}\left(\rho_{1}\right)=\left[\sum u_{i}-\sum v_{i}\right] \theta$.

We shall also need the following ring-theoretic lemma. 
Lemma 2.17 Let $R$ be a ring in which 2 is invertible, and let

$$
S=R\left[c_{1}, \ldots, c_{i}, c_{1}^{\prime}, \ldots, c_{i}^{\prime}\right]
$$

be a polynomial ring. Let $\phi: S \rightarrow S$ be the involution that exchanges $c_{j}$ and $c_{j}^{\prime}$ for all $j$. If $f \in S$ and $f+\phi(f)=0$, then $f$ may be written as a sum

$$
f=\sum_{j=1}^{i}\left(c_{j}-c_{j}^{\prime}\right) f_{j},
$$

where $\phi\left(f_{j}\right)=f_{j}$.

Proof Write $d_{j}=\frac{1}{2}\left(c_{j}-c_{j}^{\prime}\right)$ and $e_{j}=\frac{1}{2}\left(c_{j}+c_{j}^{\prime}\right)$, so that

$$
S=R\left[d_{1}, \ldots, d_{i}, e_{1}, \ldots, e_{i}\right]
$$

and that $\phi(f)=-f$ precisely when $f$ is a sum of monomial terms in which the total degree of the $d_{j}$ is odd. The result follows.

We might also be interested in the case of $R=\mathbb{Z} / 2$. Unfortunately, the polynomial $c_{1} c_{1}^{\prime} \in \mathbb{Z} / 2\left[c_{1}, c_{1}^{\prime}\right]$ furnishes a counterexample to the lemma in this case.

We now return to the proof of the proposition.

Proof First we show that the $\mathrm{E}_{2}$-page is as it is described.

Let $T_{2 n}=\left(\mathbb{G}_{m}\right)^{n} \times\left(\mathbb{G}_{m}\right)^{n}$ act on $\mathrm{GL}_{n}$ by

$$
\left(a_{1}, \ldots, a_{n}, b_{1}, \ldots, b_{n}\right) \cdot A=\operatorname{diag}\left(a_{1}, \ldots, a_{n}\right) A \operatorname{diag}\left(b_{1}^{-1}, \ldots, b_{n}^{-1}\right) .
$$

There is an evident group homomorphism $\mathbb{G}_{m} \rightarrow T_{2 n}$ given by

$$
z \mapsto\left(z^{u_{1}}, \ldots, z^{u_{n}}, z^{v_{1}}, \ldots, z^{v_{n}}\right),
$$

and for this group homomorphism, we have a commutative map of group actions

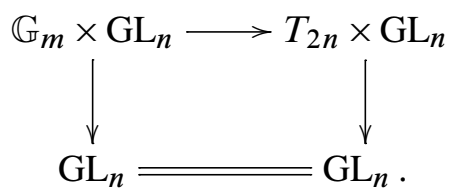

Suppose $\alpha, \beta \in \Sigma_{n}$ are each permutations on $n$-letters. Then the pair $(\alpha, \beta)$ acts on the group action $T_{2 n} \times \mathrm{GL}_{n} \rightarrow \mathrm{GL}_{n}$, where $\alpha$ permutes the first $n$ terms of $T_{2 n}$ and the columns of $\mathrm{GL}_{n}$, and $\beta$ permutes the last $n$ terms of $T_{2 n}$, and the rows of $\mathrm{GL}_{n}$. We denote this action by $f_{\alpha, \beta}$. There is also an involution, which we denote $\gamma$, which acts 
by interchanging the first and last $n$ terms of $T_{2 n}$ and is the map $A \mapsto A^{-1}$ on $\mathrm{GL}_{n}$. The identity

$$
\left[\operatorname{diag}\left(a_{1}, \ldots, a_{n}\right) A \operatorname{diag}\left(b_{1}^{-1}, \ldots, b_{n}^{-1}\right)\right]^{-1}=\operatorname{diag}\left(b_{1}, \ldots, b_{n}\right) A^{-1} \operatorname{diag}\left(a_{1}^{-1}, \ldots, a_{n}^{-1}\right)
$$

ensures that this involution is compatible with the group action.

The action of $T_{2 n}$ on $\mathrm{GL}_{n}$ yields a coaction on cohomology. We write

$$
\begin{aligned}
\mathrm{H}^{*, *}\left(T_{2 n} ; R\right) & =\Lambda_{\mathbb{M}}\left(\tau_{1}, \ldots, \tau_{n}, \tau_{1}^{\prime}, \ldots, \tau_{n}^{\prime}\right), \quad\left|\tau_{i}\right|=\left|\tau_{i}^{\prime}\right|=(1,1), \\
\mathrm{H}^{*, *}\left(\mathrm{GL}_{n} ; R\right) & =\Lambda_{\mathbb{M}}\left(\rho_{n}, \ldots, \rho_{1}\right), \quad\left|\rho_{i}\right|=(2 i-1, i) .
\end{aligned}
$$

For dimensional reasons, the coaction must be $\tau_{i} \mapsto 1 \otimes \tau_{i}$ for $i \geq 2$, but the coaction $\tau_{1}$ is more involved, and we devote the next two paragraphs to determining it.

For dimensional reasons we have

$$
\rho_{1} \mapsto 1 \otimes \rho_{1}+p_{1}\left(\tau_{1}, \ldots, \tau_{n}, \tau_{1}^{\prime}, \ldots, \tau_{n}^{\prime}\right) \otimes 1,
$$

where $p_{1}$ is a homogeneous linear polynomial. For the inclusion $\mathbb{G}_{m} \rightarrow T_{2 n}$ of the first factor, the map

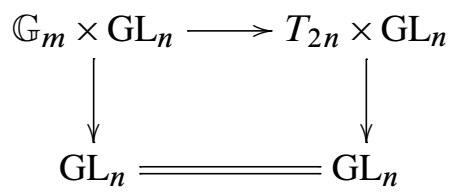

is a map of group actions. Because of the naturality of the spectral sequences and by reference to Corollary 2.9.2, we deduce that $p_{1}(\tau, 0, \ldots, 0,0, \ldots, 0)=\tau$.

On cohomology, we have $f_{\alpha, \beta}^{*}\left(\tau_{i}\right)=\tau_{\alpha^{-1}(i)}, f_{\alpha, \beta}^{*}\left(\tau_{i}^{\prime}\right)=\tau_{\beta^{-1}(i)}^{\prime}$ and $f_{\alpha, \beta}\left(\rho_{i}\right)=\rho_{i}$, the last by reference to Proposition 2.11. It follows that $p_{1}$ must be symmetric in $\left(\tau_{1}, \ldots, \tau_{n}\right)$ and $\left(\tau_{1}^{\prime}, \ldots, \tau_{n}^{\prime}\right)$, and

$$
p_{1}\left(\tau_{1}^{\prime}, \ldots, \tau_{n}^{\prime}, \tau_{1}, \ldots, \tau_{n}\right)=-p\left(\tau_{1}, \ldots, \tau_{n}, \tau_{1}^{\prime}, \ldots, \tau_{n}^{\prime}\right) .
$$

Consequently $p_{1}\left(\tau_{1}, \ldots, \tau_{n}, \tau_{1}^{\prime}, \ldots, \tau_{n}^{\prime}\right)=\sum \tau_{i}-\sum \tau_{i}^{\prime}$.

By reference to Proposition A.8, we can write down the $\mathrm{E}_{2}$-page of the spectral sequence for the $T_{2 n}$ action on $\mathrm{GL}_{n}$. We denote this by ${ }_{V} \mathrm{E}_{*}$. The $\mathrm{E}_{2}-$ page is

$$
\operatorname{Ext}_{\hat{\mathrm{H}}^{*, *}\left(T_{2 n} ; R\right)}\left(\hat{\mathrm{H}}^{*, *}\left(\mathrm{GL}_{n} ; R\right), \mathbb{M}\right)=\frac{\Lambda_{\mathbb{M}}\left(\rho_{2}, \ldots, \rho_{n}\right)\left[\theta_{1}, \ldots, \theta_{n}, \theta_{1}^{\prime}, \ldots, \theta_{n}^{\prime}\right]}{\left(\sum \theta_{i}-\sum \theta_{i}^{\prime}\right)} .
$$

The symmetric group actions give $f_{\alpha, \beta}^{*}\left(\rho_{i}\right)=\rho_{i}, f_{\alpha, \beta}^{*}\left(\theta_{i}\right)=\theta_{\alpha(i)}$ and $f_{\alpha, \beta}^{*}\left(\theta_{i}^{\prime}\right)=\theta_{\beta(i)}$. The involution acts as $\rho_{i} \mapsto-\rho_{i}, \theta_{i}=\theta_{i}^{\prime}$ and $\theta_{i}^{\prime} \mapsto \theta_{i}$. 
Now we consider which elements may support nonzero differentials, and on which pages. For dimensional reasons, powers of $\theta$ cannot support nonzero differentials, nor can $\rho_{i}$ be the image of any incoming differential. The usual arguments from Chow height and dimensions show that if $d_{i}\left(\mu \rho_{j}\right) \neq 0$ where $\mu \in \mathbb{M}^{0,0}$, then $i=j$. We will show that the differential $d_{i}\left(\rho_{i}\right)$ is as claimed.

We deduce from the symmetric group actions and the involution that

$$
d_{i}\left(\rho_{i}\right) \equiv p_{i}\left(\theta_{1}, \ldots, \theta_{n} \theta_{1}^{\prime}, \ldots, \theta_{n}^{\prime}\right)\left(\bmod \left(p_{1}, p_{2}, \ldots, p_{i-1}\right)\right),
$$

where $p_{i}$ is symmetric in the $\theta_{i}, \theta_{i}^{\prime}$ individually, and antisymmetric in the interchange of the two. In particular, writing $c_{i}$ for $\sigma_{i}\left(\theta_{i}\right)$ and $c_{i}^{\prime}$ for $\sigma_{i}\left(\theta_{i}^{\prime}\right)$, it follows from standard results on symmetric polynomials that $p_{i}$ is a polynomial in $c_{1}, \ldots, c_{n}, c_{1}^{\prime}, \ldots, c_{n}^{\prime}$.

There is map of group actions $T_{n} \times \mathrm{GL}_{n}$, being the action of the previous proposition, to the $T_{2 n} \times \mathrm{GL}_{n}$ action at hand. By comparison of the spectral sequences, it follows that $p\left(\theta_{1}, \ldots, \theta_{n}, 0, \ldots, 0\right) \equiv \sigma_{n}\left(\theta_{i}\right)$. By antisymmetry we have

$$
p_{i}\left(\theta_{1}, \ldots, \theta_{n}, \theta_{1}^{\prime}, \ldots, \theta_{n}^{\prime}\right)=\sigma_{i}\left(\theta_{i}\right)-\sigma_{i}\left(\theta_{i}^{\prime}\right)+q_{i}\left(\theta_{1}, \ldots, \theta_{n}, \theta_{1}^{\prime}, \ldots, \theta_{n}^{\prime}\right),
$$

where $q_{i}$ is of degree $i$, symmetric in $\theta_{1}, \ldots, \theta_{n}$ and $\theta_{1}^{\prime}, \ldots, \theta_{n}^{\prime}$, antisymmetric in the interchange of the $\theta_{i}$ and $\theta_{i}^{\prime}$, and $q_{i}$ lies in the product ideal

$$
I=\left(\theta_{1}, \ldots, \theta_{n}\right)\left(\theta_{1}^{\prime}, \ldots, \theta_{n}^{\prime}\right) .
$$

In terms of the $c_{i}$, we have

$$
p_{i}=c_{i}-c_{i}^{\prime}+r_{i}\left(c_{1}, \ldots, c_{i-1}, c_{1}^{\prime}, \ldots, c_{i-1}^{\prime}\right),
$$

where $r\left(c_{1}^{\prime}, \ldots, c_{i-1}^{\prime}, c_{i}, \ldots, c_{i-1}\right)=-r\left(c_{1}, \ldots, c_{i-1}, c_{i}^{\prime}, \ldots, c_{i-1}^{\prime}\right)$. By the lemma, $r$ lies in the ideal generated by $\left(c_{1}-c_{1}^{\prime}, \ldots, c_{i-1}-c_{i-1}^{\prime}\right)$. We have recursively described the differentials in the spectral sequence; they are

$$
d_{i}\left(\rho_{i}\right) \equiv c_{i}-c_{i}^{\prime}=\sigma_{i}\left(\theta_{i}\right)-\sigma_{i}\left(\theta_{i}^{\prime}\right)\left(\bmod \sigma_{1}\left(\theta_{i}\right)-\sigma_{1}\left(\theta_{i}^{\prime}\right), \ldots, \sigma_{i-1}\left(\theta_{i}\right)-\sigma_{i-1}\left(\theta_{i}^{\prime}\right)\right) .
$$

It is now a matter of no great difficulty to use our original group homomorphism $\mathbb{G}_{m} \rightarrow T_{2 n}$ to describe in full the spectral sequence for $\mathbb{G}_{m}$ acting on GL $\mathrm{L}_{n}$. We write $\mathrm{H}^{*, *}\left(\mathbb{G}_{m} ; R\right)=\Lambda_{\mathbb{M}}(\tau)$. It follows by naturality that the coaction of $\mathrm{H}^{*, *}\left(\mathbb{G}_{m} ; R\right)$ on $\mathrm{H}^{*, *}\left(\mathrm{GL}_{n} ; R\right)$ is given by $\rho_{i} \mapsto 1 \otimes \rho_{i}$ for $i \geq 2$ and $\rho_{1} \mapsto 1 \otimes \rho_{1}+\left[\sum u_{i}-\sum v_{i}\right] \tau \otimes 1$. By application of Proposition A.8, the $E_{2}$-page of the spectral sequence has the form asserted in the proposition. 
We denote this spectral sequence by ${ }_{V I} \mathrm{E}_{*}$. There is a comparison map of spectral sequences ${ }_{V} \mathrm{E}_{*} \rightarrow{ }_{V I} \mathrm{E}_{*}$, sending $\rho_{i}$ to $\rho_{i}$ for $i \geq 2$, sending $\theta_{i}$ to $u_{i} \theta$ and $\theta_{i}^{\prime}$ to $v_{i} \theta$. It follows from the comparison that in the spectral sequence ${ }_{V I} \mathrm{E}_{*}$, the differentials satisfy $d_{i}\left(\rho_{i}\right)=\left[\sigma_{i}\left(u_{i}\right)-\sigma_{i}\left(v_{i}\right)\right] \theta^{i}$, as claimed.

The argument restricting the possibilities for other nonzero differentials, and eliminating such possibilities entirely when $R$ is a field is similar to that of the previous proposition and is omitted.

Corollary 2.17.1 Let $R$ be a field of characteristic different from 2. Let $\mathbf{u}$ and $\mathbf{v}$ be two vectors in $\mathbb{Z}^{n}$, and let $\mathbb{G}_{m}$ act on $\mathrm{GL}_{n}$ by the action of (4) with weights $\mathbf{u}$ and $\mathbf{v}$. Then $\mathrm{H}^{* * *}\left(B\left(\mathbb{G}_{m}, \mathrm{GL}_{n}\right) ; R\right)$ admits the following presentation as an $\mathbb{M}_{R}$-algebra:

$\mathrm{H}^{*, *}\left(B\left(\mathbb{G}_{m}, \mathrm{GL}_{n}\right) ; R\right) \cong \frac{\Lambda_{\mathbb{M}}\left(\rho_{1}, \ldots, \rho_{2 n-1}\right)[\theta]}{I}, \quad\left|\rho_{i}\right|=(2 i-1, i), \quad|\theta|=(2,1)$,

where $I$ is generated by $\left(\theta^{j}, \rho_{j}\right)$ where $j$ is the least integer such that $\sigma_{i}(\mathbf{u}) \neq \sigma_{i}(\mathbf{v})$, or $I=0$ if there is no such $j$.

\subsection{The equivariant cohomology of Stiefel varieties}

Suppose $\mathbf{u}=\left(u_{1}, \ldots, u_{n}\right)$ is an $n$-tuple of integers. Write $f_{\mathbf{u}}(z)=\prod_{i=1}^{n}\left(z-u_{i}\right)$, a polynomial in which the coefficient of $z^{i}$ is $(-1)^{i} \sigma_{i}(\mathbf{u})$. Suppose that $\mathbf{v}=\left(v_{1}, \ldots, v_{m}\right)$ is an $m$-tuple of integers, where $m<n$. There exist polynomials $q(z)$ and $r(z)$ in $\mathbb{Q}[z]$ such that $f_{\mathbf{u}}(z)=f_{\mathbf{v}}(z) q(z)+r(z)$, where $\operatorname{deg} q(z)=n-m$ and $\operatorname{deg} r(z)<m$. In fact, since $f_{\mathbf{v}}$ is monic, the polynomials $q(z)$ and $r(z)$ have integer coefficients.

Definition 4 With notation as in the discussion above, define an approximate extension of $\mathbf{v}$ to $\mathbf{u}$ to be a vector $\operatorname{vex}_{\mathbf{u}}(\mathbf{v})=\left(v_{1}, \ldots, v_{m}, v_{m+1}^{\prime}, \ldots, v_{n}^{\prime}\right) \in \mathbb{C}^{n}$ where the $v_{i}^{\prime}$ are the roots of $q(z)$ in some order.

In general, we do not particularly care about the roots $v_{i}^{\prime}$ themselves, rather about the elementary symmetric functions of $\operatorname{vex}_{\mathbf{u}}(\mathbf{v})$. These agree up to sign with the coefficients of $f_{\mathbf{v}}(z) q(z)$, and are therefore integers. We highlight the following identity.

Lemma 2.18 With notation as above, $\sigma_{i}(\mathbf{u})-\sigma_{i}\left(\operatorname{vex}_{\mathbf{u}}(\mathbf{u})\right)$ is $(-1)^{n-i}$ times the coefficient of $z^{n-i}$ in $r(z)$. 
Theorem 2.19 Suppose the Beilinson-Soule vanishing conjecture holds for the spectrum of the ground field, Spec $k$. Let $R=\mathbb{Z}\left[\frac{1}{2}\right]$.

Let $\mathbf{u}=\left(u_{1}, \ldots, u_{n}\right) \in \mathbb{Z}^{n}$ and $\mathbf{v}=\left(v_{1}, \ldots, v_{m}\right) \in \mathbb{Z}^{m}$ be two sequences of weights with $m<n$. Consider the $\mathbb{G}_{m}$-action on $V_{m}\left(\mathbb{A}^{n}\right)$ given by

$$
z \cdot A=\operatorname{diag}\left(u_{1}, \ldots, u_{n}\right) A \operatorname{diag}\left(v_{1}^{-1}, \ldots, v_{m}^{-1}\right) .
$$

The spectral sequence computing $\mathrm{H}^{*, *}\left(B\left(\mathbb{G}_{m}, V_{m}\left(\mathbb{A}^{n}\right)\right) ; R\right)$ for the given $\mathbb{G}_{m}$-action has $\mathrm{E}_{2}$-page

$$
{ }_{I} \mathrm{E}_{2}=\operatorname{Ext}_{\widehat{\mathrm{H}}^{*, *}\left(\mathbb{G}_{m} ; R\right)}\left(\hat{\mathrm{H}}^{*, *}\left(\mathrm{GL}_{n} ; R\right), \mathbb{M}\right)=\Lambda_{\mathbb{M}}\left(\rho_{n-m+1}, \ldots, \rho_{n}\right)[\theta] .
$$

Let $n-m+1 \leq k \leq n$ and suppose that

$$
d_{j}\left(\rho_{j}\right)=0, \quad \text { for } n-m+1 \leq i<k .
$$

Then we have, in the given spectral sequence,

$$
d_{k}\left(\rho_{k}\right)=\left[\sigma_{k}(\mathbf{u})-\sigma_{k}\left(\operatorname{vex}_{\mathbf{u}}(\mathbf{v})\right)\right] \theta^{k} .
$$

Proof Strictly speaking, the proof proceeds by induction on $k \geq n-m+1$, although most of the difficulty is already evident in the base case of $k=n-m+1$. The arguments for the case $k=n-m+1$ and for the induction step are very similar; we shall give both in parallel as much as possible.

Since the cohomology of $V_{m}\left(\mathbb{A}^{n}\right)$ is $\Lambda_{\mathbb{M}}\left(\rho_{n-m+1}, \ldots, \rho_{n}\right)$ (see Corollary 2.7.1), it follows from Proposition A.7 that the $\mathrm{E}_{2}$-page is as claimed.

For dimensional reasons powers of $\theta$ cannot support a nonzero differential. From this, the product structure, and the usual argument from Chow height, it follows that the first nonvanishing differential must be of the form $d_{i}\left(\rho_{i}\right)=a \theta^{i}$ for some $i$, where $a \in \mathbb{Z}\left[\frac{1}{2}\right]$. Suppose $d_{i}\left(\rho_{i}\right)=0$ for $n-m+1 \leq i<k$, a condition that is vacuously satisfied if $k=n-m+1$. We then have $d_{k}\left(\rho_{k}\right)=a \theta^{k}$, and it remains to determine $a \in \mathbb{Z}\left[\frac{1}{2}\right]$.

Let $f_{\mathbf{u}}(z), f_{\mathbf{v}}(z)$ and $q(z)$ be as in the discussion immediately preceding the theorem. By a corollary of the Frobenius density theorem, the polynomial $q(z)$ splits over $\mathbb{Z} / p$ for infinitely many odd primes $p$. Let $P$ denote the set of all such primes.

To identify $a \in \mathbb{Z}\left[\frac{1}{2}\right]$ it suffices to calculate the class of $a$ in $\mathbb{Z} / p$ for infinitely many primes $p$, that is, it suffices to verify (5) modulo infinitely many primes $p$. We shall verify it for all primes $p \in P$.

Fix a particular prime $p \in P$. By construction there are elements $\bar{v}_{m+1}^{\prime}, \ldots, \bar{v}_{n}^{\prime}$ of $\mathbb{Z} / p$ which are roots of $q(z) \in(\mathbb{Z} / p)[z]$, and therefore there are integers $v_{m+1}^{\prime}, \ldots, v_{n}^{\prime}$ 
whose reductions are the $\bar{v}_{i}^{\prime}$. Let $\mathbf{v}^{\prime}$ denote the concatenation of $\mathbf{v}$ with these integers, viz $\mathbf{v}^{\prime}=\left(v_{1}, \ldots, v_{m}, v_{m+1}^{\prime}, \ldots, v_{n}^{\prime}\right)$.

Equip $\mathrm{GL}_{n}$ with the $\mathbb{G}_{m}$-action given by weights $\mathbf{u}$ on the left and $\mathbf{v}^{\prime}$ on the right. Then projection onto the first $m$ columns $\mathrm{GL}_{n} \rightarrow V_{m}\left(\mathbb{A}^{n}\right)$ is $\mathbb{G}_{m}$-equivariant.

When we consider the spectral sequence computing $\mathrm{H}^{* * *}\left(B\left(\mathbb{G}_{m}, \mathrm{GL}_{n}\right) ; \mathbb{Z} / p\right)$ for this action, as in Proposition 2.16, we find that $d_{i}\left(\rho_{i}\right)=\left[\sigma_{i}(\mathbf{u})-\sigma_{i}\left(\mathbf{v}^{\prime}\right)\right] \theta^{i}$. But $\sigma_{i}(\mathbf{u})$ is $(-1)^{i}$ times the coefficient of $z^{n-i}$ in $f_{\mathbf{u}}(z)$ and $\sigma_{i}\left(\mathbf{v}^{\prime}\right)$ is $(-1)^{i}$ times the coefficient of $z^{n-i}$ in $f_{\mathbf{v}}(z) q(z)$, and since $f_{\mathbf{u}}(z)=f_{\mathbf{v}}(z) q(z)+r(z)$ with $\operatorname{deg} r(z)<m$, it follows in particular that if $i<n-m$, then $d_{i}\left(\rho_{i}\right)=0$.

We continue to work with $\mathbb{Z} / p$-coefficients. For $i \geq n-m$, the class $\rho_{i}$ appears in both the spectral sequence computing $\mathrm{H}^{*, *}\left(B\left(\mathbb{G}_{m}, \mathrm{GL}_{n}\right) ; \mathbb{Z} / p\right)$ and the sequence computing $\mathrm{H}^{*, *}\left(B\left(\mathbb{G}_{m}, V_{m}\left(\mathbb{A}^{n}\right)\right) ; \mathbb{Z} / p\right)$. By comparison, if $d_{i}\left(\rho_{i}\right)=0$ for $n-m \leq i<k$ in the latter, then $d_{i}\left(\rho_{i}\right)=0$ for $n-m \leq i<k$ in the former. We find that $d_{k}\left(\rho_{k}\right)=\left[\sigma_{k}(\mathbf{u})-\sigma_{k}\left(\mathbf{v}^{\prime}\right)\right] \theta^{k}$. Observe that $\left[\sigma_{k}(\mathbf{u})-\sigma_{k}\left(\mathbf{v}^{\prime}\right)\right]$ is $(-1)^{n-k}$ times the coefficient of $z^{n-k}$ in $f_{\mathbf{u}}(z)-f_{\mathbf{v}}(z) q(z)=r(z)$, but this agrees with the reduction to $\mathbb{Z} / p$ of $\left[\sigma_{k}(\mathbf{u})-\sigma_{k}\left(\operatorname{vex}_{\mathbf{u}}(\mathbf{v})\right)\right]$ by Lemma 2.18. In particular, we have established (5) modulo $p$.

Unfortunately, this method of proof establishes only the first nonzero differential of the form $d_{k}\left(\rho_{k}\right)=C \theta^{k}$; we cannot push it further to describe the subsequent differentials. We conjecture that the pattern established in the theorem continues, that the differential takes the form

$$
d_{k}\left(\rho_{k}\right)=\left[\sigma_{k}(\mathbf{u})-\sigma_{k}\left(\operatorname{vex}_{\mathbf{u}}(\mathbf{v})\right)\right] \theta^{k},
$$

modulo the appropriate indeterminacy for all $k$.

\section{Appendix A Homological algebra}

The material in this appendix is provided to support with proof the general assertion that the spectral sequences we calculate carry the expected product structure. If the base ring were a field then all the following results would be standard, but we need them in the case where the base ring is the coefficient ring $\mathbb{M}=\mathrm{H}^{*}, *(\mathrm{pt} ; R)$, where they remain true provided one considers only finitely generated free modules, as we do.

The homological algebra we need deals with Hopf algebras over bigraded rings, graded commutative in the first grading, commutative in the second. As a convention we fix such a ring, $\mathbb{M}$. All modules will be finitely generated over $\mathbb{M}$. For an $\mathbb{M}$-module $A$ we shall write $\hat{A}$ for $\operatorname{Hom}_{\mathbb{M}}(A, \mathbb{M})$. If $A$ is free, and equipped with a distinguished 
generating set $\left\{a_{1}, \ldots, a_{n}\right\}$, we will write $\left\{\hat{a}_{1}, \ldots, \hat{a}_{n}\right\}$ for the dual generating set of $\widehat{A}$. Since we deal very often with exterior algebras, $\Lambda_{\mathbb{M}}\left(a_{1}, \ldots, a_{n}\right)$, we will decree here that such an algebra, when understood as an $\mathbb{M}$-module, should be equipped with a distinguished basis consisting of the nonzero products of the $a_{i}$.

We shall need the notion of relative Ext-groups for the $\mathbb{M}$-algebra $\hat{\mathrm{H}}^{*}, *(G)$. These can be defined via a bar construction as follows. let $N, M$ be $S$-modules, then we can form the bar complex $\perp_{*} N$ whose $p$-th term is $N \otimes_{\mathbb{M}} S^{\otimes p}$, so that

$$
\operatorname{Ext}_{S / \mathbb{M}}^{*}(N, M)=H_{*}\left(\operatorname{Hom}_{S}\left(\perp_{*} N, M\right)\right) .
$$

For the properties of such groups we refer to [23, Chapter 8]. In particular we will need the following propositions.

Proposition A.1 Let $R$ be an $\mathbb{M}$-algebra, and let

$$
0 \longrightarrow M_{1} \longrightarrow M_{2} \longrightarrow M_{3} \longrightarrow 0
$$

be a short exact sequence of $R$-modules that is split when considered as a sequence of $\mathbb{M}$-modules. Let $N$ be an $R$-module. Then there is a long exact sequence of groups

$$
\rightarrow \operatorname{Ext}_{R / \mathbb{M}}^{*}\left(M_{3}, N\right) \rightarrow \operatorname{Ext}_{R / \mathbb{M}}^{*}\left(M_{2}, N\right) \rightarrow \operatorname{Ext}_{R / \mathbb{M}}^{*}\left(M_{1}, N\right) \stackrel{\partial}{\rightarrow} \operatorname{Ext}_{R / \mathbb{M}}^{*+1}\left(M_{3}, N\right) \rightarrow
$$

Proposition A.2 Let $R_{1}, R_{2}$ be $\mathbb{M}$-algebras, with $\mathbb{M}$ graded commutative and let $M_{i}, N_{i}$ be $R_{i}$-modules for $i=1,2$. Then $M_{1} \otimes_{\mathbb{M}} M_{2}, N_{1} \otimes_{\mathbb{M}} N_{2}$ are $R_{1} \otimes_{\mathbb{M}} R_{2}-$ modules, there is an external product

$\operatorname{Ext}_{R_{1} / \mathbb{M}}^{*}\left(M_{1}, N_{1}\right) \otimes_{\mathbb{M}} \operatorname{Ext}_{R_{2} / \mathbb{M}}^{*}\left(M_{2}, N_{2}\right) \rightarrow \operatorname{Ext}_{R_{1} \otimes_{\mathbb{M}} R_{2} / \mathbb{M}}^{*}\left(M_{1} \otimes_{\mathbb{M}} M_{2}, N_{1} \otimes_{\mathbb{M}} N_{2}\right)$,

which is natural in all four variables and commutes with the connecting homomorphism of Proposition A.1.

The product arises from a standard Alexander-Whitney construction on $\perp_{*} M_{1} \otimes \perp_{*} M_{2}$.

In general we shall be dealing with group actions in the category of $s \mathbf{S h}_{\tau}(\mathbf{C})$, which is to say a group object $G$, an object $Y$, and a map $G \times Y \rightarrow Y$. The $\operatorname{ring} \widehat{S}=\widehat{\mathrm{H}}^{*}, *(G ; R)$ is therefore a Hopf algebra over $\mathbb{M}$, so there is an algebra homomorphism $\widehat{S} \rightarrow \widehat{S} \otimes_{\mathbb{M}} \widehat{S}$. Write $\widehat{N}$ for $\hat{\mathrm{H}}^{*, *}(Y ; R)$, since we shall be treating of $\operatorname{Ext}_{\widehat{S}}^{*}(\widehat{N}, \mathbb{M})$, and there is a map

$$
\widehat{N} \rightarrow \widehat{N} \otimes_{\mathbb{M}} \widehat{N}
$$

arising from the diagonal $Y \rightarrow Y \times Y$. There is a $G$-action on $Y \times Y$, via the diagonal $G \rightarrow G \times G$. The coalgebra map $\widehat{N} \rightarrow \widehat{N} \otimes_{\mathbb{M}} \widehat{N}$ is therefore $\widehat{S}$-linear. These 
conditions make $\hat{N}$ into an $\widehat{S}$-module-coalgebra. For such data, there is a product

$$
\begin{aligned}
& \operatorname{Ext}_{\hat{S} / \mathbb{M}}^{*}(\hat{N}, \mathbb{M}) \otimes_{\mathbb{M}} \operatorname{Ext}_{\hat{S} / \mathbb{M}}^{*}(\hat{N}, \mathbb{M}) \longrightarrow \operatorname{Ext}_{\hat{S} \otimes \hat{S} / \mathbb{M}}^{*}\left(\hat{N} \otimes_{\mathbb{M}} \hat{N}, \mathbb{M} \otimes_{\mathbb{M}} \mathbb{M}\right) \\
& \operatorname{Ext}_{\widehat{S} / \mathbb{M}}^{*}\left(\hat{N} \otimes_{\mathbb{M}} \hat{N}, \mathbb{M} \otimes_{\mathbb{M} \mathbb{M}) \longrightarrow}^{\longrightarrow} \operatorname{Ext}_{\hat{S} / \mathbb{M}}^{*}(\hat{N}, \mathbb{M}),\right.
\end{aligned}
$$

where the first map is the external product, and the other maps are those arising from the functoriality of $\operatorname{Ext}_{\widehat{S} / \mathbb{M}}^{*}(\widehat{N}, M)$.

We synopsize with the following.

Proposition A.3 Suppose $\widehat{S}$ is a Hopf algebra over $\mathbb{M}, \hat{N}$ is a module-coalgebra over $\widehat{S}$, then there is a natural ring structure on $\operatorname{Ext}_{\widehat{S} / \mathbb{M}}^{*}(\widehat{N}, \mathbb{M})$.

Proposition A.4 Let $\widehat{S}$ be a Hopf algebra over $\mathbb{M}$, let

$$
0 \longrightarrow \hat{N}_{1} \longrightarrow \hat{N}_{2} \longrightarrow \widehat{N}_{3} \longrightarrow 0
$$

be a short exact sequence of $\widehat{S}$-modules that splits as a sequence of $\mathbb{M}-$ modules. Then the long exact sequence of relative Ext-groups

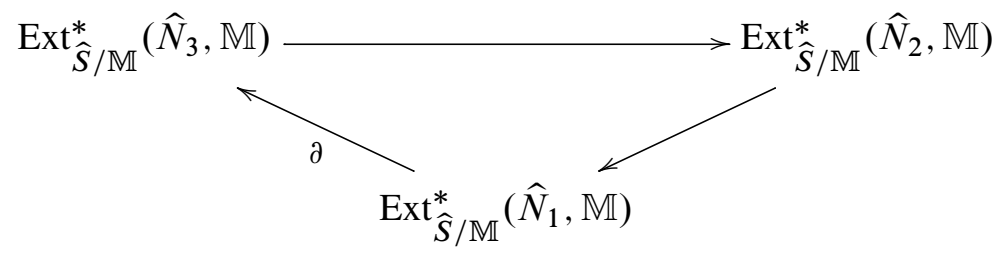

is in fact a long exact sequence of $\operatorname{Ext}_{\widehat{S} / \mathbb{M}}^{*}(\mathbb{M}, \mathbb{M})$-modules.

Proof There is a (trivial) map $\hat{N}_{i} \rightarrow \mathbb{M} \otimes_{\mathbb{M}} \widehat{N}_{i}$. Both $\widehat{N}_{i}$ and $\mathbb{M}$ are $\widehat{S}$-modules, and so we can use the external product on relative Ext-groups as before to obtain a product $\operatorname{Ext}_{\widehat{S} / \mathbb{M}}^{*}(\mathbb{M}, \mathbb{M}) \otimes_{\mathbb{M}} \operatorname{Ext}_{\widehat{S} / \mathbb{M}}^{*}\left(\hat{N}_{i}, \mathbb{M}\right)$. By Proposition A.2 the $\operatorname{Ext}_{\widehat{S} / \mathbb{M}}^{*}(\mathbb{M}, \mathbb{M})$-action is compatible with the long exact sequence of Proposition A.1.

Proposition A.5 Let $\widehat{S}$ be a Hopf algebra over $\mathbb{M}$. Let $\widehat{N}$ be an $\widehat{S}$-module coalgebra that is free as an $\mathbb{M}$-module. The ordinary Ext-group $\operatorname{Ext}_{\hat{S}}^{*}(\hat{N}, \mathbb{M})$ agrees with the relative $\operatorname{Ext}_{\widehat{S} / \mathbb{M}}^{*}(\hat{N}, \mathbb{M})$. In the case of $\hat{N}=\mathbb{M}$, the product coincides with the usual Yoneda product on $\operatorname{Ext}_{\widehat{S}}^{*}(\mathbb{M}, \mathbb{M})$. 
Proof The first assertion follows since $\perp_{*} \widehat{N}$ is a free resolution of $\widehat{N}$ when $\widehat{N}$ is free over $\mathbb{M}$.

Let $S$ be an $\mathbb{M}$-algebra. Let $D \widehat{S}$ be the derived category of bounded below complexes of $\widehat{S}$-modules. Let $A, B$ be $\widehat{S}$-modules. Recall that $\operatorname{Ext}_{\widehat{S}}^{*}(A, B)=\operatorname{Hom}_{D}(A, B)$. Observe for two $\widehat{S}$-modules $A, B$, the product $A \otimes_{\mathbb{M}} B$ is an $\widehat{S} \otimes_{\mathbb{M}} \widehat{S}$-module and consequently an $\widehat{S}$-module by restriction of scalars. It follows that $\otimes_{\mathbb{M}}$ is a right-exact bifunctor on the category of $\widehat{S}$-modules. We denote the derived version of this functor also by $\otimes_{\mathbb{M}}^{L}$. It provides us with a monoidal structure on $D \hat{S}$.

The product on $\operatorname{Ext}_{\widehat{S} / \mathbb{M}}^{*}(\widehat{N}, \mathbb{M})$ may also be constructed as the product which takes two maps in the derived category, $f, g: \widehat{N} \rightarrow \mathbb{M}$, to

$$
\widehat{N} \rightarrow \widehat{N} \otimes_{\mathbb{M}} \hat{N} \simeq \widehat{N} \otimes_{\mathbb{M}}^{L} \hat{N} \stackrel{f \otimes g}{\longrightarrow} \mathbb{M} \otimes_{\mathbb{M}} \mathbb{M} \cong \mathbb{M}
$$

In the specific case of $\widehat{N}=\mathbb{M}$, we have also a Yoneda product, which in the derived category is the composition $g \circ f: \mathbb{M} \rightarrow \mathbb{M}$. The proof that these two products coincide is standard, and is known as the Eckmann-Hilton argument.

Proposition A.6 Let $\mathbb{M}$ be a graded commutative algebra, and let

$$
S=\Lambda_{\mathbb{M}}\left(\alpha_{1}, \ldots, \alpha_{n}, \beta_{1}, \ldots, \beta_{m}\right)
$$

be a Hopf algebra over $\mathbb{M}$, with exterior algebra structure, grading given by $\left|\alpha_{i}\right|=a_{i}$ (the grading on the $\beta_{i}$ is immaterial) and coalgebra structure given by the stipulation that $\alpha_{i}, \beta_{i}$ are all primitive. Write

$$
\widehat{N}=\widehat{S} /\left(\widehat{\beta}_{1}, \ldots, \hat{\beta}_{m}\right)=\Lambda_{\mathbb{M}}\left(\widehat{\alpha}_{1}, \ldots, \widehat{\alpha}_{n}\right),
$$

which inherits a $\widehat{S}$-linear coproduct map $\widehat{N} \rightarrow \widehat{N} \otimes_{\mathbb{M}} \widehat{N}$, the action of $\widehat{S}$ on $\widehat{N} \otimes_{\mathbb{M}} \widehat{N}$ being via $\widehat{S} \rightarrow \widehat{S} \otimes_{\mathbb{M}} \widehat{S}$. There is an isomorphism of bigraded $\mathbb{M}$-algebras:

$$
\operatorname{Ext}_{\widehat{S}}^{*}(\widehat{N}, \mathbb{M}) \cong \mathbb{M}\left[\theta_{1}, \ldots, \theta_{m}\right]
$$

with $\left|\theta_{i}\right|=\left(1, a_{i}\right)$, and this isomorphism is natural in $\widehat{S}, \widehat{N}$ and $\mathbb{M}$.

Proof All tensor products are taken over $\mathbb{M}$. The naturality of the isomorphism follows from the naturality of all constructions carried out below, and we shall not mention it again.

In the case $n=0$ we have $N=\mathbb{M}$ and the result,

$$
\operatorname{Ext}_{\widehat{S}}(\mathbb{M}, \mathbb{M})=\mathbb{M}\left[\theta_{1}, \ldots, \theta_{m}\right],
$$

is well-known. 
To compute the general case of $\operatorname{Ext}_{\widehat{S}}^{*}(\widehat{N}, \mathbb{M})$, we resolve $\widehat{N}$ by a standard resolution

$$
\longrightarrow \bigoplus_{1 \leq i, j \leq n} \hat{S} \beta_{i} \beta_{j} \longrightarrow \bigoplus_{1 \leq i \leq n} \hat{S} \beta_{i} \longrightarrow \widehat{S} \longrightarrow \hat{N} .
$$

If we write $F_{j}$ for the $j$-th term; the image of the differentials lie in the submodules $\left(\beta_{1}, \ldots, \beta_{n}\right) F_{j}$. In particular, application of $\operatorname{Hom}_{\widehat{S}}(\cdot, \hat{N})$ yields a complex with trivial differential. It follows that application of $\operatorname{Hom}_{\widehat{S}}(\cdot, \mathbb{M})$ coincides with the result of applying $\operatorname{Hom}_{\widehat{S}}(\cdot, \hat{N})$, followed by $\cdot \otimes_{\widehat{N}} \mathbb{M}$. Consequently the map $\operatorname{Ext}_{\widehat{S}}^{*}(\hat{N}, \hat{N}) \rightarrow \operatorname{Ext}_{\widehat{S}}^{*}(\hat{N}, \mathbb{M})$ of $\mathbb{M}$-modules is the map

$$
\hat{N}\left[\theta_{1}, \ldots, \theta_{m}\right] \rightarrow \hat{N}\left[\theta_{1}, \ldots, \theta_{m}\right] \otimes_{\widehat{N}} \mathbb{M} \stackrel{\cong}{\longrightarrow} \mathbb{M}\left[\theta_{1}, \ldots, \theta_{m}\right] .
$$

Since this is a ring map by the naturality of the ring structure on Ext, see [23, Chapter 8], the result follows.

Proposition A.7 Let $\mathbb{M}$ be a bigraded ring, let $A$ be an exterior algebra

$$
A=\Lambda_{\mathbb{M}}\left(\alpha_{1}, \ldots, \alpha_{n}, \beta_{1}, \ldots, \beta_{m}\right)
$$

that is also a Hopf algebra with the elements $\alpha_{i}, \beta_{i}$ primitive and homogeneous. Let $B$ be a ring

$$
B=\Lambda_{R}\left(\alpha_{1}^{\prime}, \ldots, \alpha_{n}^{\prime}, \gamma_{1}, \ldots, \gamma_{p}\right)
$$

with $\alpha_{i}^{\prime}, \gamma_{i}$ homogeneous which is equipped with a comodule structure over $A$, where $B \rightarrow A \otimes_{\mathbb{M}} B$ is given by

$$
\alpha_{i}^{\prime} \mapsto \alpha_{i} \otimes 1+1 \otimes \alpha_{i}^{\prime}, \quad \gamma_{i} \mapsto 1 \otimes \gamma_{i} .
$$

Let $\hat{A}$ denote the dual algebra of $A$ over $\mathbb{M}$, and $\widehat{B}$ the dual coalgebra over $\mathbb{M}$. Then $\widehat{B}$ is an $\hat{A}$-module, and there is an isomorphism

$$
\operatorname{Ext}_{\widehat{A}}^{*}(\widehat{B}, \mathbb{M})=\Lambda_{\mathbb{M}}\left(\gamma_{1}^{\prime}, \ldots, \gamma_{p}^{\prime}\right)\left[\theta_{1}, \ldots, \theta_{m}\right],
$$

which is again natural in $\widehat{A}, \widehat{B}, \mathbb{M}$. There is a map

$$
\operatorname{Ext}_{\widehat{A}}^{*}(\widehat{B}, \mathbb{M}) \rightarrow \operatorname{Ext}_{\mathbb{M}}^{*}(\widehat{B}, \mathbb{M})=B,
$$

mapping $\gamma_{i}^{\prime}$ to $\gamma_{i}$. The element $\theta_{i}$ corresponds to $\beta_{i}$; if $\beta_{i}$ has bidegree $(r, s)$, then $\theta_{i}$ has bidegree $(r, s)$ in $\operatorname{Ext}_{\widehat{A}}^{1}(\hat{B}, \mathbb{M})$.

Proof All constructions and isomorphisms are natural. Since $B$ is free and finitely generated as an $\mathbb{M}$-module, it follows that $\operatorname{Hom}_{\mathbb{M}}(B, \mathbb{M})=\widehat{B}$ is too. Consequently,

$$
\operatorname{Ext}_{\mathbb{M}}^{*}(\widehat{B}, \mathbb{M})=\operatorname{Hom}_{\mathbb{M}}(\widehat{B}, \mathbb{M})=\widehat{\hat{B}}=B
$$


For a subset $J \subset\{1, \ldots, p\}$, write $\gamma_{J}$ for the product $\prod_{i \in J} \gamma_{i}$. We can decompose $\widehat{B}$ as a direct sum indexed over products:

$$
\bigoplus_{J \subset\{1, \ldots, p\}} \frac{\hat{A}}{\left(\hat{\beta}_{1}, \ldots, \hat{\beta}_{m}\right)} \hat{\gamma}_{J}
$$

With the given decomposition and by use of the previous proposition, we have

$\operatorname{Ext}_{\hat{A}}^{*}(\widehat{B}, \mathbb{M})=\underset{J \subset\{1, \ldots, p\}}{\bigoplus} \operatorname{Ext}_{\widehat{A}}^{*}\left(\frac{\hat{A}}{\left(\hat{\beta}_{1}, \ldots, \hat{\beta}_{m}\right)}, \mathbb{M}\right) \hat{\widehat{\gamma}}_{J}=\bigoplus_{J \subset\{1, \ldots, p\}} \mathbb{M}\left[\theta_{1}, \ldots, \theta_{m}\right] \widehat{\hat{\gamma}}_{J}$.

The indeterminates $\theta_{i}$ lie in $\operatorname{Ext}_{\widehat{A}}^{1}(\widehat{B}, \mathbb{M})$. What remains to be determined is the multiplication $\hat{\hat{\gamma}}_{J} \widehat{\hat{\gamma}}_{J^{\prime}}$, but the ring map $\operatorname{Ext}_{\widehat{A}}^{*}(\widehat{B}, \mathbb{M}) \rightarrow B$ takes $\hat{\gamma}_{i}$ in the former to $\gamma_{i}$ in the latter, and since

$$
\widehat{\hat{\gamma}}_{i} \in \operatorname{Ext}_{\widehat{A}}^{0}(\widehat{B}, \mathbb{M})=\bigoplus_{J \subset\{1, \ldots, p\}} \mathbb{M} \widehat{\hat{\gamma}}_{J}
$$

it follows that $\widehat{\hat{\gamma}_{i}} \widehat{\hat{\gamma}}_{j}=\widehat{\widehat{\gamma_{i} \gamma_{j}}}$. We are therefore justified in dropping the distinction and write $\hat{\hat{\gamma}}_{i}=\gamma_{i}$.

We must account for one additional complexity in our calculation of Ext-rings.

Proposition A.8 Let $\mathbb{M}$ be a bigraded ring, let $A$ be an exterior algebra

$$
A=\Lambda_{\mathbb{M}}\left(\alpha_{1}, \ldots, \alpha_{n}, \beta_{1}, \ldots, \beta_{m}\right)
$$

that is also a Hopf algebra where the elements $\alpha_{i}, \beta_{i}$ are primitive and homogeneous. Let $B$ be a ring

$$
B=\Lambda_{\mathbb{M}}\left(\alpha_{1}^{\prime}, \ldots, \alpha_{n}^{\prime}, \gamma_{1}, \ldots, \gamma_{p}, \eta\right),
$$

where the elements $\alpha_{i}^{\prime}, \gamma_{i}$ and $\eta$ are homogeneous, and which is equipped with a comodule structure over $A$, where $B \rightarrow A \otimes_{\mathbb{M}} B$ is given by

$$
\alpha_{i}^{\prime} \mapsto \alpha_{i} \otimes 1+1 \otimes \alpha_{i}^{\prime}, \quad \gamma_{i} \mapsto 1 \otimes \gamma_{i}, \quad \eta \mapsto 1 \otimes \eta+\sum_{i=1}^{m} b_{i} \beta_{i} \otimes 1,
$$

where $b_{1}, \ldots, b_{m} \in \mathbb{M}^{0,0}$. Let $\hat{A}$ denote the dual algebra of $A$ over $\mathbb{M}$, and $\hat{B}$ the dual coalgebra over $\mathbb{M}$. Then $\hat{B}$ is an $\hat{A}$-module, and there is an exact sequence of 
trigraded $\Lambda_{\mathbb{M}}\left(\gamma_{1}^{\prime}, \ldots, \gamma_{p}^{\prime}\right)\left[\theta_{1}, \ldots, \theta_{m}\right]$-modules

$$
\begin{aligned}
0 \rightarrow \frac{\Lambda_{\mathbb{M}}\left(\gamma_{1}^{\prime}, \ldots, \gamma_{p}^{\prime}\right)\left[\theta_{1}, \ldots, \theta_{m}\right]}{\left(\sum b_{i} \theta_{i}\right)} \rightarrow \operatorname{Ext}_{\hat{A}}^{*}(\widehat{B}, \mathbb{M}) & \\
& \rightarrow \operatorname{Ann}_{\Lambda_{\mathbb{M}}\left(\gamma_{1}^{\prime}, \ldots, \gamma_{p}^{\prime}\right)\left[\theta_{1}, \ldots, \theta_{m}\right]}\left(\sum b_{i} \theta_{i}\right) \rightarrow 0 .
\end{aligned}
$$

There is a natural map

$$
\operatorname{Ext}_{\widehat{A}}^{*}(\widehat{B}, \mathbb{M}) \rightarrow \operatorname{Ext}_{\mathbb{M}}^{*}(\widehat{B}, \mathbb{M})=B,
$$

mapping $\gamma_{i}^{\prime}$ to $\gamma_{i}$. The element $\theta_{i}$ corresponds to $\beta_{i}$; if $\beta_{i}$ has bidegree $(r, s)$, then $\theta_{i}$ has bidegree $(r, s)$ in $\operatorname{Ext}_{\hat{A}}^{1}(\widehat{B}, \mathbb{M})$.

Proof As before, we can reduce the problem by decomposing the $\hat{A}$-module $\widehat{B}$ into direct summands generated by monomials in the $\hat{\gamma}_{i}$. We therefore assume that $p=0$.

We define the ring $\widehat{S}=\Lambda_{\mathbb{M}}\left(\widehat{\alpha}_{1}, \ldots, \widehat{\alpha}_{m}\right)$, which is an $\widehat{A}$-algebra. There is an isomorphism $\widehat{B}=\widehat{S} \oplus \widehat{S} \hat{\eta}$, where the $\widehat{S}$-module-structure is the evident one and $\hat{\beta}_{i} \cdot 1=b_{i} \hat{\eta}$.

The map $\mathbb{M} \rightarrow \widehat{S}$ induces the evident map

$$
\mathbb{M}\left[\theta_{1}, \ldots, \theta_{m}\right] \cong \operatorname{Ext}_{\widehat{A}}(\widehat{S}, \mathbb{M}) \rightarrow \operatorname{Ext}_{\widehat{A}}(\mathbb{M}, \mathbb{M})=\mathbb{M}\left[\theta_{1}, \ldots, \theta_{m}, \phi_{1}, \ldots, \phi_{n}\right],
$$

where the $\theta_{i}$ correspond to the $\beta_{i}$ and the $\phi_{i}$ to the $\alpha_{i}$.

There is a short exact sequence of $\hat{A}$-modules which splits as a sequence of $\widehat{S}$-modules:

$$
0 \longrightarrow \widehat{S} \stackrel{1 \mapsto \widehat{\eta}}{\longrightarrow} \widehat{B} \longrightarrow \widehat{S} \longrightarrow 0 .
$$

From this one obtains a long exact sequence of Ext-groups which is a sequence of $\operatorname{Ext}_{\widehat{A}}^{*}(\mathbb{M}, \mathbb{M})-$-modules, and so of $\operatorname{Ext}_{\widehat{A}}^{*}(\widehat{S}, \mathbb{M})$-modules:

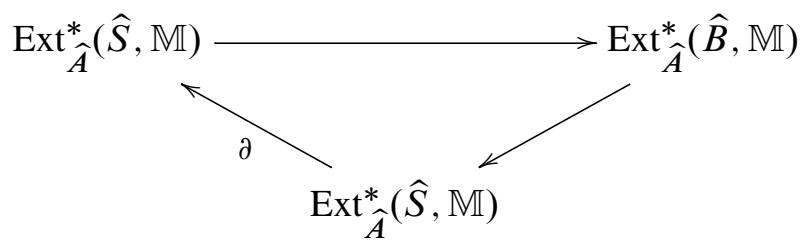

By explicit calculation in the snake lemma, the boundary map

$$
\operatorname{Hom}_{\widehat{A}}(\widehat{S}, \mathbb{M})=\mathbb{M} \rightarrow \operatorname{Ext}_{\widehat{A}}^{1}(\widehat{S}, \mathbb{M})=\mathbb{M}\left[\theta_{1}, \ldots, \theta_{m}\right]^{(1)}
$$


takes 1 to $\sum b_{i} \theta_{i}$. The long exact sequence now gives the exact sequence

$$
\begin{aligned}
& \longrightarrow \operatorname{Ext}_{\hat{A}}^{*}(\widehat{S}, \mathbb{M}) \stackrel{1 \mapsto \sum b_{i} \theta_{i}}{\longrightarrow} \operatorname{Ext}_{\hat{A}}^{*}(\widehat{S}, \mathbb{M}) \rightarrow \operatorname{Ext}_{\hat{A}}^{*}(\widehat{B}, \mathbb{M}) \rightarrow \operatorname{Ext}_{\widehat{A}}^{*}(\widehat{S}, \mathbb{M}) \rightarrow \\
& \begin{array}{cc}
\downarrow & \downarrow \cong \\
\mathbb{M}\left[\theta_{1}, \ldots, \theta_{m}\right] & \mathbb{M}\left[\theta_{1}, \ldots, \theta_{m}\right]
\end{array}
\end{aligned}
$$

and this is in fact an exact sequence of $\operatorname{Ext}_{\widehat{A}}^{*}(\widehat{S}, \mathbb{M})$-modules, by Proposition A.4, so $\operatorname{Ext}_{\hat{A}}^{*}(\widehat{B}, \mathbb{M})$ fits into a short exact sequence:

$$
0 \longrightarrow \frac{\mathbb{M}\left[\theta_{1}, \ldots, \theta_{m}\right]}{\left(\sum b_{i} \theta_{i}\right)} \longrightarrow \operatorname{Ext}_{\widehat{A}}^{*}(\hat{B}, \mathbb{M}) \longrightarrow \operatorname{Ann}_{\mathbb{M}\left[\theta_{1}, \ldots, \theta_{m}\right]}\left(\sum b_{i} \theta_{i}\right) \longrightarrow 0 .
$$

This is the claim of the proposition in the case $p=0$.

Corollary A.8.1 In the notation of the preceding problem, if each $b_{i}$ is invertible in $\mathbb{M}^{0,0}$, then the $\mathrm{E}_{2}$-page takes the form

$$
\frac{\Lambda_{\mathbb{M}}\left(\gamma_{1}^{\prime}, \ldots, \gamma_{p}^{\prime}\right)\left[\theta_{1}, \ldots, \theta_{m}\right]}{\left(\sum b_{i} \theta_{i}\right)} \cong \operatorname{Ext}_{\widehat{A}}^{*}(\widehat{B}, R) .
$$

Proof Under the hypotheses given, the element $\sum b_{i} \theta_{i}$ is not a zero divisor in the $\operatorname{ring} \Lambda_{\mathbb{M}}\left(\gamma_{1}^{\prime}, \ldots, \gamma_{p}^{\prime}\right)\left[\theta_{1}, \ldots, \theta_{m}\right]$.

Acknowledgements This paper has been too long in the writing, and the author is certain that many people have helped him in ways that he has gracelessly forgotten. He wishes to thank in particular Gunnar Carlsson for introducing him to motivic cohomology and the fiber-to-base spectral sequence; he also wishes to thank Eric Friedlander for telling him the folklore of the Beilinson-Soulé vanishing conjecture and Anssi Lahtinen for many stimulating conversations.

\section{References}

[1] S Bloch, Algebraic cycles and higher K-theory, Adv. in Math. 61 (1986) 267-304 MR852815

[2] J M Boardman, Conditionally convergent spectral sequences, from: "Homotopy invariant algebraic structures", (J-P Meyer, J Morava, W S Wilson, editors), Contemp. Math. 239, Amer. Math. Soc., Providence, RI (1999) 49-84 MR1718076

[3] A K Bousfield, D M Kan, Homotopy limits, completions and localizations, Lecture Notes in Mathematics 304, Springer, Berlin (1972) MR0365573 
[4] D Dugger, D C Isaksen, Motivic cell structures, Algebr. Geom. Topol. 5 (2005) 615652 MR2153114

[5] D Edidin, W Graham, Equivariant intersection theory, Invent. Math. 131 (1998) 595-634 MR1614555

[6] H Esnault, B Kahn, M Levine, E Viehweg, The Arason invariant and mod 2 algebraic cycles, J. Amer. Math. Soc. 11 (1998) 73-118 MR1460391

[7] A Grothendieck, Torsion homologique et sections rationnelles, from: "Séminaire Claude Chevalley (2e année, 1958): Anneaux de Chow et applications", volume 3, Secrétariat mathématique, Paris (1958) MR0110704

[8] PS Hirschhorn, Model categories and their localizations, Mathematical Surveys and Monographs 99, American Mathematical Society (2003) MR1944041

[9] D C Isaksen, Flasque model structures for simplicial presheaves, $K$-Theory 36 (2005) 371-395 MR2275013

[10] J F Jardine, Simplicial presheaves, J. Pure Appl. Algebra 47 (1987) 35-87 MR906403

[11] J P May, Classifying spaces and fibrations, Mem. Amer. Math. Soc. 1 (1975) xiii+98 MR0370579

[12] J P May, A concise course in algebraic topology, University of Chicago Press (1999) MR1702278

[13] C Mazza, V Voevodsky, C Weibel, Lecture notes on motivic cohomology, Clay Mathematics Monographs 2, American Mathematical Society (2006) MR2242284

[14] F Morel, V Voevodsky, $\mathbf{A}^{1}$-homotopy theory of schemes, Inst. Hautes Études Sci. Publ. Math. (1999) 45-143 MR1813224

[15] Y A Nisnevich, The completely decomposed topology on schemes and associated descent spectral sequences in algebraic $K$-theory, from: "Algebraic $K$-theory: Connections with geometry and topology", (J F Jardine, V P Snaith, editors), NATO Adv. Sci. Inst. Ser. C Math. Phys. Sci. 279, Kluwer Acad. Publ., Dordrecht (1989) 241-342 MR1045853

[16] O Pushin, Higher Chern classes and Steenrod operations in motivic cohomology, K-Theory 31 (2004) 307-321 MR2068875

[17] M Rothenberg, NE Steenrod, The cohomology of classifying spaces of $H$-spaces, Bull. Amer. Math. Soc. 71 (1965) 872-875 MR0208596

[18] G Segal, Classifying spaces and spectral sequences, Inst. Hautes Études Sci. Publ. Math. (1968) 105-112 MR0232393

[19] B Totaro, The Chow ring of a classifying space, from: "Algebraic $K$-theory", (W Raskind, C Weibel, editors), Proc. Sympos. Pure Math. 67, Amer. Math. Soc., Providence, RI (1999) 249-281 MR1743244 
[20] A Vistoli, On the cohomology and the Chow ring of the classifying space of $\mathrm{PGL}_{p}, \mathrm{~J}$. Reine Angew. Math. 610 (2007) 181-227 MR2359886

[21] V Voevodsky, Reduced power operations in motivic cohomology, Publ. Math. Inst. Hautes Études Sci. (2003) 1-57 MR2031198

[22] V Voevodsky, Motivic Eilenberg-Maclane spaces, Publ. Math. Inst. Hautes Études Sci. (2010) 1-99 MR2737977

[23] C A Weibel, An introduction to homological algebra, Cambridge Studies in Advanced Mathematics 38, Cambridge Univ. Press (1994) MR1269324

[24] B Williams, The motivic cohomology of Stiefel varieties, Journal of $K$-Theory 10 (2012) 141-163 MR2990564

Department of Mathematics, University of Southern California

Kaprielian Hall, 3620 South Vermont Avenue, Los Angeles, CA 90089-2532, USA

tbwillia@usc.edu

Received: 28 March 2012 Revised: 2 August 2012 